\title{
On some short-distance properties of the fourth-rank hadronic vacuum polarization tensor and the anomalous magnetic moment of the muon
}

\section{Marc Knecht}

Centre de Physique Théorique, CNRS/Aix-Marseille Univ./Univ. de Toulon (UMR 7332), CNRS-Luminy Case 907, 13288 Marseille Cedex 9, France

E-mail: knecht@cpt.univ-mrs.fr

ABSTRACT: Some short-distance properties of the fourth-rank hadronic vacuum polarization tensor are re-examined. Their consequences are critically discussed in the context of the hadronic light-by-light scattering contribution to the anomalous magnetic moment of the muon.

Keywords: QCD Phenomenology

ARXIV EPRINT: 2005.09929 


\section{Contents}

1 Introduction 1

2 Some hadronic four- and three-point functions and their properties 3

2.1 Some properties of the rank-four hadronic vacuum polarization tensor $\mathcal{W}_{\mu \nu \rho \sigma} \quad 4$

2.2 Properties of the three-point function $\mathcal{W}_{\mu \nu \rho} \quad 5$

3 Implementing the short-distance constraints $\quad 8$

3.1 The pion pole 9

$\begin{array}{lll}3.2 & \text { An apparent paradox and its solution } & 12\end{array}$

$\begin{array}{lll}3.3 & \text { Pseudoscalar poles } & 14\end{array}$

4 Summary and conclusion $\quad 15$

$\begin{array}{ll}\text { A Technical aspects of the short-distance expansion } & 17\end{array}$

B The low-energy expansion of the three-point function $\mathcal{W}_{\mu \nu \rho} \quad 20$

\section{Introduction}

The Muon g-2 Collaboration is about to release, some 15 years after the final publication [1] of the BNL-E821 experiment, the first result on a new high-precision measurement, conducted by the FNAL-E989 experiment, of the anomalous magnetic moment of the muon $a_{\mu}$. It is thus not surprising that quite some theoretical activity aiming at improving the standard model prediction for this observable is going on. The main limitations on this endeavour come from the hadronic contributions, hadronic vacuum polarization (HVP) and hadronic light-by-light scattering (HLxL). The former is traditionally evaluated through a dispersion relation, whose absorptive part is determined directly from data on $e^{+} e^{-} \rightarrow$ hadrons. The most recent evaluations [2-4] along these lines have now reached a precision that, in relative terms, lies below the $0.5 \%$ level. The determination of HVP from numerical simulations of QCD on a lattice has been developing fast in recent years. Recent results [5-10], although they have not yet reached the same level of precision than the traditional approach, look promising. Finally, the MUonE proposal [11], which aims at an experimental evaluation of HVP directly in the space-like region, and in an inclusive manner, could be an interesting complementary alternative for the future, although both theoretical and experimental challenges are high [12-14].

Assuming that the results for the HVP contribution obtained through these various approaches will eventually agree and reach comparable precisions, HLxL will then stand out as the main source of theoretical uncertainty, hence the many recent efforts devoted 
to its evaluation. Here also, various approaches are being considered and developed, see ref. [15] for a thorough and up-to-date review, as well as for an extensive list of references. Reducing the theoretical relative uncertainty of the HLxL contribution to a reliable level of $\sim 10 \%$ would already constitute a remarkable achievement.

This note is devoted to some aspects related to one of the specific contributions to HLxL, namely the one due to the pion pole. There are essentially two reasons that explain why this particular contribution to HLxL has attracted so much attention in the past, and keeps on being a point of focus even today. First, in the limit where the number of colours $N_{c}$ becomes large [16], only single-meson exchanges are relevant [17], and the pion being the lightest meson, it is expected to provide the main contribution [18, 19]. Second, in the first serious attempts [20-26] to perform a complete evaluation of the HLxL contribution to $a_{\mu}$, it so occurred that the final result was in fact almost completely given by the contribution due to the pion pole, the other contributions cancelling almost exactly among themselves. This cancellation took place although the hadronic models considered in the various studies were actually exhibiting quite different features, as discussed, for instance, in ref. [27]. Thus, having the pion-pole contribution under good control is currently considered to be an essential step into the direction of obtaining an accurate and reliable evaluation of the HLxL component of $a_{\mu}$.

In the past various authors $[20-24,28,29]$ have used different definitions of what they have chosen to call the contribution from the "pion pole", see the discussion in the review [30]. At present, this issue does no longer seem to constitute a point of contention. Recently a different debate concerning the pion-pole contribution to HLxL has resurfaced in the literature [31-33]. It has been triggered mainly because of different views as how to implement a certain short-distance constraint, first obtained in ref. [34], on the rank-four hadronic vacuum polarization tensor, which is the central object for HLxL. These different views then lead to quite different numerical evaluations of the pion-pole contribution, see for instance the discussion in ref. [32]. Although the pion pole is only one contribution to HLxL among many, and what should actually matter in the end is the full contribution to $a_{\mu}$ from HLxL, it is certainly of interest, given the importance of this contribution, to understand what are the whys and wherefores of this rather confusing situation.

Thus, the purpose of this note is therefore not to provide yet another new evaluation of the HLxL contribution. Rather, it was written with the aim of scrutinizing this particular issue in greater detail and, possibly, of providing some understanding that may contribute to settle it. The outline of the remaining part of the text is as follows. First, I recall, in section 2, general properties of the four- and three-point functions relevant for this discussion, including the short-distance condition that relates them. I then describe in detail the implementation of this condition in section 3 in general, before focusing on its implications for the contribution of the pseudoscalar poles. Finally, I give a summary and conclusions in section 4. Some more technical aspects related to the short-distance expansion have been gathered in appendix A for the interested reader. Appendix B illustrates the discussion from the perspective of the low-energy expansion. The contents of these appendices are, to the best of my knowledge, not available from the already existing literature, and may thus have some interest by themselves. 


\section{Some hadronic four- and three-point functions and their properties}

As mentioned in the introduction, the central object of interest is the connected four-point QCD correlator

$$
\begin{aligned}
& \mathcal{W}_{\mu \nu \rho \sigma}\left(q_{1}, q_{2}, q_{3}, q_{4}\right) \\
& \quad=\frac{1}{i} \int d^{4} x_{1} \int d^{4} x_{2} \int d^{4} x_{3} e^{i\left(q_{1} \cdot x_{1}+q_{2} \cdot x_{2}+q_{3} \cdot x_{3}\right)}\left\langle\Omega\left|T\left\{j_{\mu}\left(x_{1}\right) j_{\nu}\left(x_{2}\right) j_{\rho}\left(x_{3}\right) j_{\sigma}(0)\right\}_{C}\right| \Omega\right\rangle,
\end{aligned}
$$

where $j_{\mu}(x)$ stands for the light-quark component of the hadronic part of the electromagnetic current,

$$
j_{\mu}=\bar{\psi} Q \gamma_{\mu} \psi, \psi=\left(\begin{array}{c}
u \\
d \\
s
\end{array}\right), Q=\operatorname{diag}\left(+\frac{2}{3},-\frac{1}{3},-\frac{1}{3}\right),
$$

and $|\Omega\rangle$ denotes the QCD vacuum. For notational convenience, I have written this correlator as a function of four variables, but only three momenta are actually independent, since invariance under translations requires that the condition

$$
q_{1}+q_{2}+q_{3}+q_{4}=0
$$

holds. Let me recall that $a_{\mu}^{\mathrm{HLxL}}$, the HLxL contribution to the anomalous magnetic moment of the muon, can be expressed in terms of this correlator in the following way [35]

$$
a_{\mu}^{\mathrm{HLxL}} \equiv \frac{1}{48 m} \operatorname{tr}\left[(\not p+m)\left[\gamma^{\sigma}, \gamma^{\tau}\right](\not p+m) \Gamma_{\sigma \tau}^{\mathrm{HLxL}}(p, p)\right]
$$

where $p$ and $m$ stand for the momentum and the mass of the muon, respectively, and $\Gamma_{\sigma \tau}^{\mathrm{HLx}}(p, p)$ is the limit of the vertex function $\Gamma_{\sigma \tau}^{\mathrm{HLxL}}\left(p^{\prime}, p\right)$, defined as

$$
\begin{aligned}
\overline{\mathrm{u}}\left(p^{\prime}\right) \Gamma_{\sigma \tau}^{\mathrm{HL} x \mathrm{~L}}\left(p^{\prime}, p\right) \mathrm{u}(p)= & e^{6} \int \frac{d^{4} q_{1}}{(2 \pi)^{4}} \int \frac{d^{4} q_{2}}{(2 \pi)^{4}}, \frac{1}{q_{1}^{2} q_{2}^{2}\left(q_{1}+q_{2}-k\right)^{2}} \\
& \times \frac{1}{\left(p^{\prime}-q_{1}\right)^{2}-m^{2}} \frac{1}{\left(p^{\prime}-q_{1}-q_{2}\right)^{2}-m^{2}} \\
& \times \overline{\mathrm{u}}\left(p^{\prime}\right) \gamma^{\mu}\left(\not p^{\prime}-\not q_{1}+m\right) \gamma^{\nu}\left(\not p^{\prime}-q_{1}-\not q_{2}+m\right) \gamma^{\rho} \mathrm{u}(p) \\
& \times \mathcal{W}_{\mu \nu \rho \sigma \tau}\left(q_{1}, q_{2}, k-q_{1}-q_{2},-k\right),
\end{aligned}
$$

when the momentum difference $k=p^{\prime}-p$ vanishes. This definition involves the derivative of the four-point function,

$$
\mathcal{W}_{\mu \nu \rho \sigma \tau}\left(q_{1}, q_{2}, k-q_{1}-q_{2},-k\right) \equiv \frac{\partial}{\partial k^{\sigma}} \mathcal{W}_{\mu \nu \rho \tau}\left(q_{1}, q_{2}, k-q_{1}-q_{2},-k\right)
$$

with respect to its fourth momentum $k$. Eq. (2.4) then requires to take the limit $k \rightarrow 0$ of this derivative. I next recall some properties of this four-point function relevant for the present discussion. 


\subsection{Some properties of the rank-four hadronic vacuum polarization tensor $\mathcal{W}_{\mu \nu \rho \sigma}$}

Due to the conservation of the current $j_{\mu}$, the rank-four hadronic vacuum polarization tensor satisfies the Ward identities

$$
\left\{q_{1 \mu} ; q_{2 \nu} ; q_{3 \rho} ; q_{4 \sigma}\right\} \mathcal{W}^{\mu \nu \rho \sigma}\left(q_{1}, q_{2}, q_{3}, q_{4}\right)=\{0 ; 0 ; 0 ; 0\}
$$

Based on these transversality properties combined with Bose symmetry, the authors of ref. [36] have obtained a decomposition of the tensor $\mathcal{W}^{\mu \nu \rho \tau}$,

$$
\mathcal{W}^{\mu \nu \rho \tau}\left(q_{1}, q_{2}, q_{3}, q_{4}\right)=\sum_{i=1}^{54} \mathcal{W}_{i}\left(q_{1}, q_{2}, q_{3}, q_{4}\right) T_{i}^{\mu \nu \rho \tau}\left(q_{1}, q_{2}, q_{3}, q_{4}\right),
$$

in terms of invariant functions $\mathcal{W}_{i}\left(q_{1}, q_{2}, q_{3}, q_{4}\right)$ free from kinematic singularities and zeros. These functions actually depend on the invariants that can be built with the products $q_{i} \cdot q_{j}, i, j=1,2,3,4$, but for simplicity I write them as functions of the momenta for the time being. Not much is known about these functions beyond the kinematic properties mentioned above, and in order to estimate them, or at least the subset of them that contributes to $a_{\mu}^{\mathrm{HLL}}$, it is important to make sure that they satisfy the few properties that can be deduced directly from QCD. One of these properties arises from the well-known behaviour $[37,38]$ of the time-ordered product of two currents $(2.2)$ at short distances, ${ }^{1}$

$$
\lim _{q \rightarrow \infty} \int d^{4} x e^{i q \cdot x} T\left\{j_{\mu}(x) j_{\nu}(0)\right\}=-2 \epsilon_{\mu \nu \alpha \beta} \frac{q^{\alpha}}{q^{2}} A^{\beta}(0)+\mathcal{O}\left(q^{-2}\right),
$$

where it is understood here and in what follows that the limit holds when the momentum $q$ belongs to the Euclidean region and when all its components become simultaneously large. The axial current appearing on the right-hand side of this relation is defined as

$$
A_{\mu} \equiv \bar{\psi} Q^{2} \gamma_{\mu} \gamma_{5} \psi=\sum_{a=3,8,0} \operatorname{tr}\left(Q^{2} \lambda^{a}\right) A_{\mu}^{a}, A_{\mu}^{a} \equiv \bar{\psi} \frac{\lambda^{a}}{2} \gamma_{\mu} \gamma_{5} \psi, \frac{\lambda^{0}}{2} \equiv \frac{\mathbb{1}}{\sqrt{6}} .
$$

Then, writing

$$
q_{1}=\bar{q}+\hat{q}, q_{2}=\bar{q}-\hat{q}, \hat{q}^{2}=-Q^{2}, Q^{2}>0,
$$

one establishes [34] the following short-distance behaviour when the momenta carried by the first two currents become hard, while the other two remain soft (note that $q_{3}+q_{4}=-q_{1}-q_{2}$ remains soft as well),

$$
\mathcal{W}_{\mu \nu \rho \sigma}\left(\bar{q}+\hat{q}, \bar{q}-\hat{q}, q_{3}, q_{4}\right)=-2 \epsilon_{\mu \nu \tau \alpha} \frac{\hat{q}^{\alpha}}{\hat{q}^{2}} \mathcal{W}_{\rho \sigma}^{\tau}\left(q_{3}, q_{4}\right)+\mathcal{O}\left(\hat{q}^{-2}\right)
$$

where the right-hand side involves the three-point function $\mathcal{W}_{\rho \sigma}{ }^{\tau}\left(q_{3}, q_{4}\right)$ constructed with two electromagnetic currents $j_{\rho}, j_{\sigma}$, and the axial current $A^{\tau}$. Its properties are discussed in the following sub-section.

\footnotetext{
${ }^{1}$ There are also constraints on $\mathcal{W}^{\mu \nu \rho \tau}\left(q_{1}, q_{2}, q_{3}, q_{4}\right)$ arising from the short-distance behaviour of the time-ordered product of three currents. These are discussed in ref. [39], but will not be considered here.
} 


\subsection{Properties of the three-point function $\mathcal{W}_{\mu \nu \rho}$}

The three-point function $\mathcal{W}_{\mu \nu \rho}\left(q_{1}, q_{2}\right)$ is defined as

$$
\mathcal{W}_{\mu \nu \rho}\left(q_{1}, q_{2}\right)=i \int d^{4} x_{1} \int d^{4} x_{2} e^{i\left(q_{1} \cdot x_{1}+q_{2} \cdot x_{2}\right)}\left\langle\Omega\left|T\left\{j_{\mu}\left(x_{1}\right) j_{\nu}\left(x_{2}\right) A_{\rho}(0)\right\}\right| \Omega\right\rangle .
$$

It satisfies the Ward identities

$$
\left\{q_{1}^{\mu} ; q_{2}^{\nu}\right\} \mathcal{W}_{\mu \nu \rho}\left(q_{1}, q_{2}\right)=\{0 ; 0\},\left(q_{1}+q_{2}\right)^{\rho} \mathcal{W}_{\mu \nu \rho}\left(q_{1}, q_{2}\right)=\mathcal{A} \epsilon_{\mu \nu \alpha \beta} q_{1}^{\alpha} q_{2}^{\beta}+\mathcal{W}_{\mu \nu}\left(q_{1}, q_{2}\right)
$$

where $\mathcal{A}$ stands for the anomalous contribution [40, 41]

$$
\mathcal{A}=-\frac{N_{c}}{2 \pi^{2}} \operatorname{tr} Q^{4}=-\frac{N_{c}}{9 \pi^{2}}
$$

These Ward identities feature yet another three-point function,

$$
\mathcal{W}_{\mu \nu}\left(q_{1}, q_{2}\right) \equiv \int d^{4} x_{1} \int d^{4} x_{2} e^{i\left(q_{1} \cdot x_{1}+q_{2} \cdot x_{2}\right)}\left\langle\operatorname{vac}\left|T\left\{j_{\mu}\left(x_{1}\right) j_{\nu}\left(x_{2}\right)\left[D(0)+\frac{\alpha_{s}}{6 \pi}(G \cdot \widetilde{G})(0)\right]\right\}\right| \operatorname{vac}\right\rangle,
$$

with

$$
D \equiv \bar{\psi}\left\{Q^{2}, \mathcal{M}\right\} i \gamma_{5} \psi, \mathcal{M}=\operatorname{diag}\left(m_{u}, m_{d}, m_{s}\right),(G \cdot \widetilde{G}) \equiv \frac{1}{2} \epsilon_{\mu \nu \rho \sigma} G^{\mu \nu} G^{\rho \sigma},
$$

where $m_{q}, q=u, d, s$, denotes the masses of the three lightest quarks and $G^{\mu \nu}$ is the gluon field strength. The decomposition of $\mathcal{W}_{\mu \nu}\left(q_{1} ; q_{2}\right)$ is quite simple, since it involves a single function that is also free of kinematic singularities,

$$
\mathcal{W}_{\mu \nu}\left(q_{1}, q_{2}\right)=\mathcal{H}\left(q_{1}^{2}, q_{2}^{2},\left(q_{1}+q_{2}\right)^{2}\right) \epsilon_{\mu \nu \alpha \beta} q_{1}^{\alpha} q_{2}^{\beta}
$$

This representation is entirely fixed by Lorentz covariance, Bose symmetry, invariance under parity and conservation of the current $j_{\mu}$, which imposes transversality,

$$
\left\{q_{1}^{\mu} ; q_{2}^{\nu}\right\} \mathcal{W}_{\mu \nu}\left(q_{1}, q_{2}\right)=\{0 ; 0\}
$$

Achieving a similar decomposition for the three-point function $\mathcal{W}_{\mu \nu \rho}\left(q_{1}, q_{2}\right)$ is not quite as straightforward. Using only Lorentz covariance, invariance under parity, Bose symmetry and Schouten's identity to eliminate two additional possible structures, namely $q_{1}^{\nu} \epsilon^{\mu \rho \alpha \beta} q_{1 \alpha} q_{2 \beta}+q_{2}^{\mu} \epsilon^{\nu \rho \alpha \beta} q_{1 \alpha} q_{2 \beta}$ and $q_{2}^{\nu} \epsilon^{\mu \rho \alpha \beta} q_{1 \alpha} q_{2 \beta}-q_{1}^{\mu} \epsilon^{\nu \rho \alpha \beta} q_{1 \alpha} q_{2 \beta}$, one obtains, to start with, the general decomposition

$$
\begin{aligned}
\mathcal{W}_{\mu \nu \rho}\left(q_{1}, q_{2}\right)= & \epsilon_{\mu \nu \alpha \beta} q_{1}^{\alpha} q_{2}^{\beta}\left(q_{1}+q_{2}\right)_{\rho} W_{0}\left(q_{1}^{2}, q_{2}^{2},\left(q_{1}+q_{2}\right)^{2}\right) \\
& +\epsilon_{\mu \nu \alpha \beta} q_{1}^{\alpha} q_{2}^{\beta}\left(q_{1}-q_{2}\right)_{\rho} W_{1}\left(q_{1}^{2}, q_{2}^{2},\left(q_{1}+q_{2}\right)^{2}\right) \\
& +\left[q_{1 \nu} \epsilon_{\mu \rho \alpha \beta} q_{1}^{\alpha} q_{2}^{\beta}-q_{2 \mu} \epsilon_{\nu \rho \alpha \beta} q_{1}^{\alpha} q_{2}^{\beta}\right] W_{2}\left(q_{1}^{2}, q_{2}^{2},\left(q_{1}+q_{2}\right)^{2}\right) \\
& +\left[q_{1 \mu} \epsilon_{\nu \rho \alpha \beta} q_{1}^{\alpha} q_{2}^{\beta}+q_{2 \nu} \epsilon_{\mu \rho \alpha \beta} q_{1}^{\alpha} q_{2}^{\beta}\right] W_{3}\left(q_{1}^{2}, q_{2}^{2},\left(q_{1}+q_{2}\right)^{2}\right) \\
& +\epsilon_{\mu \nu \rho \alpha}\left(q_{1}-q_{2}\right)^{\alpha} W_{4}\left(q_{1}^{2}, q_{2}^{2},\left(q_{1}+q_{2}\right)^{2}\right)+\epsilon_{\mu \nu \rho \alpha}\left(q_{1}+q_{2}\right)^{\alpha} W_{5}\left(q_{1}^{2}, q_{2}^{2},\left(q_{1}+q_{2}\right)^{2}\right) .
\end{aligned}
$$


in terms of six amplitudes that are free of kinematic singularities. The use of Schouten's identity, as well as Bose symmetry, may well introduce kinematic zeros, but this issue is not relevant for our present purposes, so I will not take it into consideration. Bose symmetry further requires

$$
W_{i}\left(q_{2}^{2}, q_{1}^{2},\left(q_{1}+q_{2}\right)^{2}\right)=(-1)^{i} W_{i}\left(q_{1}^{2}, q_{2}^{2},\left(q_{1}+q_{2}\right)^{2}\right), i=0,1,2,3,4,5 .
$$

Conservation of the electromagnetic current implies

$$
W_{5}\left(q_{1}^{2}, q_{2}^{2},\left(q_{1}+q_{2}\right)^{2}\right)+\left(q_{1}^{2}+q_{2}^{2}\right) W_{3}\left(q_{1}^{2}, q_{2}^{2},\left(q_{1}+q_{2}\right)^{2}\right)=0,
$$

and

$2 W_{4}\left(q_{1}^{2}, q_{2}^{2},\left(q_{1}+q_{2}\right)^{2}\right)-\left(q_{1}^{2}-q_{2}^{2}\right) W_{3}\left(q_{1}^{2}, q_{2}^{2},\left(q_{1}+q_{2}\right)^{2}\right)+2\left(q_{1} \cdot q_{2}\right) W_{2}\left(q_{1}^{2}, q_{2}^{2},\left(q_{1}+q_{2}\right)^{2}\right)=0$.

These identities allow to eliminate $W_{4}\left(q_{1}^{2}, q_{2}^{2},\left(q_{1}+q_{2}\right)^{2}\right)$ and $W_{5}\left(q_{1}^{2}, q_{2}^{2},\left(q_{1}+q_{2}\right)^{2}\right)$ in terms of the remaining functions without introducing kinematic singularities. The result reads

$$
\mathcal{W}^{\mu \nu \rho}\left(q_{1}, q_{2}\right)=\sum_{i=0}^{3} W_{i}\left(q_{1}^{2}, q_{2}^{2},\left(q_{1}+q_{2}\right)^{2}\right) \tau_{i}^{\mu \nu \rho}\left(q_{1}, q_{2}\right)
$$

with

$$
\begin{aligned}
\tau_{0}^{\mu \nu \rho}\left(q_{1}, q_{2}\right) & =\epsilon^{\mu \nu \alpha \beta} q_{1 \alpha} q_{2 \beta}\left(q_{1}+q_{2}\right)^{\rho}, \\
\tau_{1}^{\mu \nu \rho}\left(q_{1}, q_{2}\right) & =\epsilon^{\mu \nu \alpha \beta} q_{1 \alpha} q_{2 \beta}\left(q_{1}-q_{2}\right)^{\rho}, \\
\tau_{2}^{\mu \nu \rho}\left(q_{1}, q_{2}\right) & =q_{1}^{\nu} \epsilon^{\mu \rho \alpha \beta} q_{1 \alpha} q_{2 \beta}-q_{2}^{\mu} \epsilon^{\nu \rho \alpha \beta} q_{1 \alpha} q_{2 \beta}-\left(q_{1} \cdot q_{2}\right) \epsilon^{\mu \nu \rho \alpha}\left(q_{1}-q_{2}\right)_{\alpha}, \\
\tau_{3}^{\mu \nu \rho}\left(q_{1}, q_{2}\right) & =q_{1}^{\mu} \epsilon^{\nu \rho \alpha \beta} q_{1 \alpha} q_{2 \beta}+q_{2}^{\nu} \epsilon^{\mu \rho \alpha \beta} q_{1 \alpha} q_{2 \beta}-q_{1}^{2} \epsilon^{\mu \nu \rho \alpha} q_{2 \alpha}-q_{2}^{2} \epsilon^{\mu \nu \rho \alpha} q_{1 \alpha} .
\end{aligned}
$$

An alternative but equivalent decomposition in terms of four functions free of kinematic singularities can also be found in ref. [42] or in eq. (4.9) of ref. [43]. The condition (2.14) on $\left(q_{1}+q_{2}\right)^{\rho} \mathcal{W}_{\mu \nu \rho}\left(q_{1} ; q_{2}\right)$ further requires

$$
\begin{aligned}
& \left(q_{1}+q_{2}\right)^{2}\left[W_{0}\left(q_{1}^{2}, q_{2}^{2},\left(q_{1}+q_{2}\right)^{2}\right)+W_{2}\left(q_{1}^{2}, q_{2}^{2},\left(q_{1}+q_{2}\right)^{2}\right)\right] \\
+ & \left(q_{1}^{2}-q_{2}^{2}\right)\left[W_{1}\left(q_{1}^{2}, q_{2}^{2},\left(q_{1}+q_{2}\right)^{2}\right)-W_{3}\left(q_{1}^{2}, q_{2}^{2},\left(q_{1}+q_{2}\right)^{2}\right)\right] \\
- & \left(q_{1}^{2}+q_{2}^{2}\right) W_{2}\left(q_{1}^{2}, q_{2}^{2},\left(q_{1}+q_{2}\right)^{2}\right)-\mathcal{A}-\mathcal{H}\left(q_{1}^{2}, q_{2}^{2},\left(q_{1}+q_{2}\right)^{2}\right)=0,
\end{aligned}
$$

when combined with eq. (2.18). Expressing $W_{0}\left(q_{1}^{2}, q_{2}^{2},\left(q_{1}+q_{2}\right)^{2}\right)$ in terms of the remaining functions through this relation leads to the decomposition given in ref. [44], with a slightly different notation,

$$
\begin{aligned}
\mathcal{W}_{\mu \nu \rho}\left(q_{1}, q_{2}\right)= & \frac{\left(q_{1}+q_{2}\right)_{\rho}}{\left(q_{1}+q_{2}\right)^{2}}\left[\mathcal{A}+\mathcal{H}\left(q_{1}^{2}, q_{2}^{2},\left(q_{1}+q_{2}\right)^{2}\right)\right] \epsilon_{\mu \nu \alpha \beta} q_{1}^{\alpha} q_{2}^{\beta} \\
& +\sum_{i=1}^{3} w_{i}\left(q_{1}^{2}, q_{2}^{2},\left(q_{1}+q_{2}\right)^{2}\right) t_{i}^{\mu \nu \rho}\left(q_{1}, q_{2}\right)
\end{aligned}
$$


in terms of a set of three fully transverse tensors $t_{i}^{\mu \nu \rho}\left(q_{1}, q_{2}\right)$,

$$
\begin{aligned}
& t_{1}^{\mu \nu \rho}\left(q_{1}, q_{2}\right)=\tau_{1}^{\mu \nu \rho}\left(q_{1}, q_{2}\right)-\frac{q_{1}^{2}-q_{2}^{2}}{\left(q_{1}+q_{2}\right)^{2}} \tau_{0}^{\mu \nu \rho}\left(q_{1}, q_{2}\right), \\
& t_{2}^{\mu \nu \rho}\left(q_{1}, q_{2}\right)=\tau_{2}^{\mu \nu \rho}\left(q_{1}, q_{2}\right)-\tau_{0}^{\mu \nu \rho}\left(q_{1}, q_{2}\right)+\frac{q_{1}^{2}+q_{2}^{2}}{\left(q_{1}+q_{2}\right)^{2}} \tau_{0}^{\mu \nu \rho}\left(q_{1}, q_{2}\right), \\
& t_{3}^{\mu \nu \rho}\left(q_{1}, q_{2}\right)=\tau_{1}^{\mu \nu \rho}\left(q_{1}, q_{2}\right)+\tau_{3}^{\mu \nu \rho}\left(q_{1}, q_{2}\right),
\end{aligned}
$$

and with

$$
\begin{aligned}
& w_{1}\left(q_{1}^{2}, q_{2}^{2},\left(q_{1}+q_{2}\right)^{2}\right)=W_{1}\left(q_{1}^{2}, q_{2}^{2},\left(q_{1}+q_{2}\right)^{2}\right)-W_{3}\left(q_{1}^{2}, q_{2}^{2},\left(q_{1}+q_{2}\right)^{2}\right), \\
& w_{2}\left(q_{1}^{2}, q_{2}^{2},\left(q_{1}+q_{2}\right)^{2}\right)=W_{2}\left(q_{1}^{2}, q_{2}^{2},\left(q_{1}+q_{2}\right)^{2}\right), \\
& w_{3}\left(q_{1}^{2}, q_{2}^{2},\left(q_{1}+q_{2}\right)^{2}\right)=W_{3}\left(q_{1}^{2}, q_{2}^{2},\left(q_{1}+q_{2}\right)^{2}\right) .
\end{aligned}
$$

But this elimination is done at the expense of introducing kinematic singularities into the tensors $t_{i}^{\mu \nu \rho}\left(q_{1}, q_{2}\right)$, and hence a kinematic constraint on the functions $w_{i}\left(q_{1}^{2}, q_{2}^{2},\left(q_{1}+q_{2}\right)^{2}\right)$. Indeed, eq. (2.26) precisely materializes this constraint, since it states that the combination

$$
\left(q_{1}^{2}+q_{2}^{2}\right) w_{2}\left(q_{1}^{2}, q_{2}^{2},\left(q_{1}+q_{2}\right)^{2}\right)-\left(q_{1}^{2}-q_{2}^{2}\right) w_{1}\left(q_{1}^{2}, q_{2}^{2},\left(q_{1}+q_{2}\right)^{2}\right)+\mathcal{A}+\mathcal{H}\left(q_{1}^{2}, q_{2}^{2},\left(q_{1}+q_{2}\right)^{2}\right)
$$

has to be equal to $\left(q_{1}+q_{2}\right)^{2}$ times a function free of any kinematic singularity.

Since the authors of ref. [32] use the notation of ref. [44], let me, before closing this section, provide the connection between the two. It is straightforward to establish the relations

$t^{(+) \mu \nu \rho}\left(q_{1}, q_{2}\right)=t_{2}^{\mu \nu \rho}\left(q_{1}, q_{2}\right), \quad t^{(-) \mu \nu \rho}\left(q_{1}, q_{2}\right)=t_{1}^{\mu \nu \rho}\left(q_{1}, q_{2}\right), \quad \tilde{t}^{(-) \mu \nu \rho}\left(q_{1}, q_{2}\right)=t_{3}^{\mu \nu \rho}\left(q_{1}, q_{2}\right)$,

Then, upon writing [44]

$$
\begin{aligned}
\mathcal{W}_{\mu \nu \rho}\left(q_{1}, q_{2}\right)=-\frac{1}{8 \pi^{2}} & {\left[-w_{L}\left(q_{1}, q_{2}\right) \tau_{0 \mu \nu \rho}\left(q_{1}, q_{2}\right)+w_{T}^{(+)}\left(q_{1}, q_{2}\right) t_{\mu \nu \rho}^{(+)}\left(q_{1}, q_{2}\right)\right.} \\
+ & \left.w_{T}^{(-)}\left(q_{1}, q_{2}\right) t_{\mu \nu \rho}^{(-)}\left(q_{1}, q_{2}\right)+\tilde{w}_{T}^{(-)}\left(q_{1}, q_{2}\right) \tilde{t}_{\mu \nu \rho}^{(-)}\left(q_{1}, q_{2}\right)\right],
\end{aligned}
$$

one obtains

$$
\begin{aligned}
& 8 \pi^{2} w_{1}\left(q_{1}, q_{2}\right)=-w_{T}^{(-)}\left(q_{1}, q_{2}\right), \quad 8 \pi^{2} w_{2}\left(q_{1}, q_{2}\right)=-w_{T}^{(+)}\left(q_{1}, q_{2}\right), \\
& 8 \pi^{2} w_{3}\left(q_{1}, q_{2}\right)=-\tilde{w}_{T}^{(-)}\left(q_{1}, q_{2}\right),
\end{aligned}
$$

and, making, for convenience, the change of notation $W_{0} \longrightarrow w_{0}$,

$$
\begin{aligned}
\frac{\left(q_{1}+q_{2}\right)^{2}}{8 \pi^{2}} w_{L}\left(q_{1}, q_{2}\right)= & \left(q_{1}+q_{2}\right)^{2}\left[w_{0}\left(q_{1}, q_{2}\right)+w_{2}\left(q_{1}, q_{2}\right)\right] \\
& +\left(q_{1}^{2}-q_{2}^{2}\right) w_{1}\left(q_{1}, q_{2}\right)-\left(q_{1}^{2}+q_{2}^{2}\right) w_{2}\left(q_{1}, q_{2}\right) \\
= & \mathcal{A}+\mathcal{H}\left(q_{1}^{2}, q_{2}^{2},\left(q_{1}+q_{2}\right)^{2}\right) .
\end{aligned}
$$

It is clear from this relation that the function $w_{L}\left(q_{1}^{2}, q_{2}^{2},\left(q_{1}+q_{2}\right)^{2}\right)$ does in general exhibit kinematic singularities. At this stage, let me formulate two remarks: 
- All the above properties still hold if instead of considering the correlators involving the current $A_{\mu}$, I had replaced the latter by one of its components $A_{\mu}^{a}$ defined in eq. (2.10), with the proviso that each function like $W_{i}$ or $w_{i}$ is endowed with a corresponding superscript $a$, where $a=3,8,0$, and that the anomalous contribution $\mathcal{A}$ is replaced by $\mathcal{A}^{a} \equiv \mathcal{A} \operatorname{tr}\left(Q^{2} \lambda^{a} / 2\right) / \operatorname{tr} Q^{4}$. Following common practice, I will refer to these three cases $a=3,8,0$ as the iso-triplet, octet, and singlet channels, respectively.

- In the limit where $q_{2}$ vanishes, or equivalently in the combined limit $q_{2}^{2} \rightarrow 0,\left(q_{1}+\right.$ $\left.q_{2}\right)^{2} \rightarrow q_{1}^{2}$, the relation $(2.34)$ becomes

$$
\frac{1}{8 \pi^{2}} w_{L}\left(q_{1}^{2}, 0, q_{1}^{2}\right)=w_{0}\left(q_{1}^{2}, 0, q_{1}^{2}\right)+w_{1}\left(q_{1}^{2}, 0, q_{1}^{2}\right)=\frac{1}{q_{1}^{2}}\left[\mathcal{A}+\mathcal{H}\left(q_{1}^{2}, 0, q_{1}^{2}\right)\right] .
$$

Two observations can be made from this relation. The first is that the combination $\mathcal{A}+\mathcal{H}\left(q_{1}^{2}, 0, q_{1}^{2}\right)$ vanishes as $\mathcal{O}\left(q_{1}^{2}\right)$, a statement in which, when restricted to the iso-triplet channel and with the anomaly removed, one recognizes the SutherlandVeltman theorem [45, 46], see also refs. [43, 47]. The second observation is more relevant for the subject of this note: in the chiral limit, or in the combined chiral and large- $N_{c}$ limit in the case of the singlet channel, $\mathcal{H}\left(q_{1}^{2}, 0, q_{1}^{2}\right)$ vanishes, and the relation (2.35) reduces to the usual expression $w_{L}\left(q_{1}^{2}, 0, q_{1}^{2}\right) / 8 \pi^{2}=\mathcal{A} / q_{1}^{2}$. Although the combination $w_{0}\left(q_{1}^{2}, 0, q_{1}^{2}\right)+w_{1}\left(q_{1}^{2}, 0, q_{1}^{2}\right)$ tends to the same expression in this limit, the way it arises, and the physical content it conveys, is completely different. I will come back to this issue and its consequences later on.

Finally, let me mention that the first equality in eq. (2.34) also appears as eq. (B13) of ref. [48], but its implications have not been discussed by the authors of this article.

\section{Implementing the short-distance constraints}

Coming back to the short-distance behaviour given in eq. (2.12), it may now be rewritten as

$$
\mathcal{W}^{\mu \nu \rho \sigma}\left(\bar{q}+\hat{q}, \bar{q}-\hat{q}, q_{3}, q_{4}\right)=-\frac{2}{\hat{q}^{2}} \sum_{i=0}^{3} w_{i}\left(q_{3}^{2}, q_{4}^{2},\left(q_{3}+q_{4}\right)^{2}\right) K_{i}^{\mu \nu \rho \sigma}\left(\hat{q}, q_{3}, q_{4}\right)+\mathcal{O}\left(\hat{q}^{-2}\right),
$$

where

$$
\begin{aligned}
& K_{i}^{\mu \nu \rho \sigma}\left(\hat{q}, q_{3}, q_{4}\right) \equiv \epsilon^{\mu \nu \tau \alpha} \hat{q}_{\alpha} \tau_{i}^{\rho \sigma}{ }_{\tau}\left(q_{3}, q_{4}\right) \quad i=0,1,2, \\
& K_{3}^{\mu \nu \rho \sigma}\left(\hat{q}, q_{3}, q_{4}\right) \equiv \epsilon^{\mu \nu \tau \alpha} \hat{q}_{\alpha}\left[\tau_{1}^{\rho \sigma}{ }_{\tau}\left(q_{3}, q_{4}\right)+\tau_{3}^{\rho \sigma}{ }_{\tau}\left(q_{3}, q_{4}\right)\right] .
\end{aligned}
$$

The task that needs to be done next is to work out the consequences of the short-distance constraint (3.1) on the invariant functions $\mathcal{W}_{i}\left(q_{1}, q_{2}, q_{3}, q_{4}\right)$ that describe the fourth-rank vacuum polarization tensor as shown in eq. (2.8). A procedure through which this can be achieved is described in appendix A. Here I will merely discuss, through one example, some of the consequences that follow from the condition (3.1). 
The example I wish to consider involves, following ref. [49], the combination

$$
\hat{\mathcal{W}}_{1}\left(q_{1}, q_{2}, q_{3}, q_{4}\right) \equiv \mathcal{W}_{1}\left(q_{1}, q_{2}, q_{3}, q_{4}\right)-\left(q_{1} \cdot q_{2}\right) \mathcal{W}_{47}\left(q_{1}, q_{2}, q_{3}, q_{4}\right) .
$$

For this combination, which satisfies $\hat{\mathcal{W}}_{1}\left(q_{1}, q_{2}, q_{3}, q_{4}\right)=\hat{\mathcal{W}}_{1}\left(q_{2}, q_{1}, q_{3}, q_{4}\right)$, the shortdistance constraint (3.1) requires the condition

$$
\hat{\mathcal{W}}_{1}\left(\bar{q}+\hat{q}, \bar{q}-\hat{q}, q_{3}, q_{4}\right)=-\frac{2}{\hat{q}^{2}}\left[w_{0}\left(q_{3}^{2}, q_{4}^{2},\left(q_{3}+q_{4}\right)^{2}\right)+w_{1}\left(q_{3}^{2}, q_{4}^{2},\left(q_{3}+q_{4}\right)^{2}\right)\right]+\mathcal{O}\left(\hat{q}^{-4}\right)
$$

to hold. Before considering some specific aspects of this relation, a few general statements may be useful:

- This condition holds as it stands, i.e. for all values of the invariants $q_{3}^{2}, q_{4}^{2}$ and $\left(q_{3}+q_{4}\right)^{2}$.

- Both sides are free from kinematic singularities. Since such singularities are absent on the left-hand side by construction, none should show up on the right-hand side, which is the case.

- Dynamical singularities in the variables $q_{3}^{2}, q_{4}^{2}$ and $\left(q_{3}+q_{4}\right)^{2}$, i.e. poles due to singleparticle exchanges or cuts due to multi-particle exchanges, have to match on both sides; those present in the functions $w_{0}$ and $w_{1}$ must correspond to singularities also present in $\hat{\mathcal{W}}_{1}$ and that moreover survive in the limit under consideration; likewise, singularities in $\hat{\mathcal{W}}_{1}$ that have no counterpart in $w_{0}$ or $w_{1}$ must fall into the sub-leading contributions to the short-distance expansion.

- Since the momenta $q_{3}$ and $q_{4}$ are generic (i.e. non-exceptional in the sense of Weinberg's theorem [50]), the chiral limit can be taken on both sides; the same holds for the large- $N_{c}$ limit, or for the combination of both limits.

- The limit where in addition $q_{3}^{2}$ becomes large in the Euclidean region can also be taken on both sides, as long as the condition $-\hat{q}^{2} \gg-q_{3}^{2}$ remains satisfied.

\subsection{The pion pole}

Let us now consider the contribution coming from the exchange of a single neutral pion. It produces in $\hat{\mathcal{W}}_{1}$ a pole in the variable $\left(q_{3}+q_{4}\right)^{2}$,

$$
\hat{\mathcal{W}}_{1}^{\left(\pi^{0}\right)}\left(q_{1}, q_{2}, q_{3}, q_{4}\right)=-\frac{\mathcal{F}_{\pi \gamma^{*} \gamma^{*}}\left(q_{1}^{2}, q_{2}^{2}\right) \mathcal{F}_{\pi \gamma^{*} \gamma^{*}}\left(q_{3}^{2}, q_{4}^{2}\right)}{\left(q_{3}+q_{4}\right)^{2}-M_{\pi}^{2}}
$$

involving the pion transition form factor $\mathcal{F}_{\pi \gamma^{*} \gamma^{*}}$ defined as

$$
i \int d^{4} x e^{i q \cdot x}\left\langle\Omega\left|T\left\{j_{\mu}(x / 2) j_{\nu}(-x / 2)\right\}\right| \pi^{0}(p)\right\rangle=\epsilon_{\mu \nu \alpha \beta} q^{\alpha} p^{\beta} \mathcal{F}_{\pi \gamma^{*} \gamma^{*}}\left((p / 2+q)^{2},(p / 2-q)^{2}\right),
$$

and where Bose symmetry means that the form factor is unchanged upon replacing $q$ by $-q$. Notice that the above definition implies that

$$
\lim _{q \rightarrow \pm p / 2} \mathcal{F}_{\pi \gamma^{*} \gamma^{*}}\left((p / 2+q)^{2},(p / 2-q)^{2}\right)=\mathcal{F}_{\pi \gamma^{*} \gamma^{*}}\left(M_{\pi}^{2}, 0\right)=\mathcal{F}_{\pi \gamma^{*} \gamma^{*}}\left(0, M_{\pi}^{2}\right)
$$


It differs of course from

$$
\lim _{(p / 2 \pm q)^{2} \rightarrow 0} \mathcal{F}_{\pi \gamma^{*} \gamma^{*}}\left((p / 2+q)^{2},(p / 2-q)^{2}\right)=\mathcal{F}_{\pi \gamma^{*} \gamma^{*}}\left(M_{\pi}^{2} / 2+2 q^{2}, 0\right)=\mathcal{F}_{\pi \gamma^{*} \gamma^{*}}\left(0, M_{\pi}^{2} / 2+2 q^{2}\right) .
$$

A pole singularity similar to the one in $\hat{\mathcal{W}}_{1}$ also shows up on the right-hand side of eq. (3.4), since

$$
w_{0}\left(q_{3}^{2}, q_{4}^{2},\left(q_{3}+q_{4}\right)^{2}\right)=\operatorname{tr}\left(Q^{2} \lambda^{3}\right) \frac{F_{\pi} \mathcal{F}_{\pi \gamma^{*} \gamma^{*}}\left(q_{3}^{2}, q_{4}^{2}\right)}{\left(q_{3}+q_{4}\right)^{2}-M_{\pi}^{2}}+\cdots
$$

where the ellipsis stands for terms that are regular at $\left(q_{3}+q_{4}\right)^{2}=M_{\pi}^{2}$ and $F_{\pi}$ denotes the pion decay constants defined as

$$
\left\langle\Omega\left|A_{\mu}^{3}(0)\right| \pi^{0}(p)\right\rangle=i F_{\pi} p_{\mu} .
$$

That the pion pole is located in the function $w_{0}$ and that it takes the form given above follows directly from the structure of the three-point function $\mathcal{W}^{\mu \nu \rho}$ as given in eq. (2.24), and from the structure of the two matrix elements in eqs. (3.6) and (3.10). The interested reader may actually check this property explicitly on the calculation of the functions $w_{i}$ at next-to-leading order in the low-energy expansion presented in appendix B. According to the third item in the list that follows eq. (3.4), this same pole singularity in $w_{0}$ has to be recovered in the asymptotic limit of $\hat{\mathcal{W}}_{1}\left(\bar{q}+\hat{q}, \bar{q}-\hat{q}, q_{3}, q_{4}\right)$. This requires

$$
\lim _{-\hat{q}^{2} \rightarrow+\infty} \mathcal{F}_{\pi \gamma^{*} \gamma^{*}}\left(\hat{q}^{2}, \hat{q}^{2}\right)=\frac{2}{3} \frac{F_{\pi}}{\hat{q}^{2}}+\mathcal{O}\left(\hat{q}^{-4}\right),
$$

a property that is known to hold [51, 52], and that also follows from the result given in eq. (2.9). Furthermore, the compatibility, via the short-distance constraint (3.4), between the two expressions (3.5) and (3.9) manifestly continues to hold in the chiral limit.

We may now consider the kinematic regime relevant for the evaluation of $a_{\mu}^{\mathrm{HLxL}}$. According to the formulas given in eqs. (2.4), (2.5), and (2.6), this involves taking the derivative of the rank-four vacuum polarization tensor with respect to $q_{4}$, and then letting $q_{4} \rightarrow 0$, taking the constraint (2.3) into account. Since the tensors $T_{i}^{\mu \nu \rho \sigma}\left(q_{1}, q_{2}, q_{3}, q_{4}\right)$ are all at least linear in the momentum $q_{4}$, this limit can be rewritten as

$$
\begin{aligned}
\lim _{q_{4} \rightarrow 0} \frac{\partial}{\partial q_{4}^{\sigma}} \mathcal{W}_{\mu \nu \rho \tau}\left(q_{1}, q_{2},-q_{4}-q_{1}-q_{2}, q_{4}\right) \equiv & \left.\sum_{i} \mathcal{W}_{i}\left(q_{1}, q_{2}, q_{3}, q_{4}\right)\right|_{q_{4}=0} \\
& \times \lim _{q_{4} \rightarrow 0} \frac{\partial}{\partial q_{4}^{\sigma}} T_{i}^{\mu \nu \rho \tau}\left(q_{1}, q_{2},-q_{4}-q_{1}-q_{2}, q_{4}\right) .
\end{aligned}
$$

As far as the short-distance constraint (3.4) is concerned, this means that we need to compare the leading term in the short-distance expansion of

$$
\begin{aligned}
\lim _{q_{4} \rightarrow 0}-\frac{\mathcal{F}_{\pi \gamma^{*} \gamma^{*}}\left((\bar{q}+\hat{q})^{2},(\bar{q}-\hat{q})^{2}\right) \mathcal{F}_{\pi \gamma^{*} \gamma^{*}}\left(q_{3}^{2}, q_{4}^{2}\right)}{\left(q_{3}+q_{4}\right)^{2}-M_{\pi}^{2}} & =-\frac{\mathcal{F}_{\pi \gamma^{*} \gamma^{*}}\left((\bar{q}+\hat{q})^{2},(\bar{q}-\hat{q})^{2}\right) \mathcal{F}_{\pi \gamma^{*} \gamma^{*}}\left(M_{\pi}^{2}, 0\right)}{q_{3}^{2}-M_{\pi}^{2}} \\
& =-\frac{2}{3} \frac{F_{\pi}}{\hat{q}^{2}} \frac{\mathcal{F}_{\pi \gamma^{*} \gamma^{*}}\left(M_{\pi}^{2}, 0\right)}{q_{3}^{2}-M_{\pi}^{2}}+\mathcal{O}\left(\hat{q}^{-4}\right)
\end{aligned}
$$


with

$$
\lim _{q_{4} \rightarrow 0}-\frac{2}{\hat{q}^{2}} \operatorname{tr}\left(Q^{2} \lambda^{3}\right) \frac{F_{\pi} \mathcal{F}_{\pi \gamma^{*} \gamma^{*}}\left(q_{3}^{2}, q_{4}^{2}\right)}{\left(q_{3}+q_{4}\right)^{2}-M_{\pi}^{2}}=-\frac{2}{\hat{q}^{2}} \operatorname{tr}\left(Q^{2} \lambda^{3}\right) \frac{F_{\pi} \mathcal{F}_{\pi \gamma^{*} \gamma^{*}}\left(M_{\pi}^{2}, 0\right)}{q_{3}^{2}-M_{\pi}^{2}} .
$$

The two expressions clearly match, and keep on doing so if one further takes the chiral limit, where one gains the additional information that $\mathcal{F}_{\pi} \mathcal{F}_{\pi \gamma^{*} \gamma^{*}}\left(M_{\pi}^{2}, 0\right) \rightarrow F_{0} \stackrel{\circ}{\mathcal{F}}_{\pi \gamma^{*} \gamma^{*}}(0,0)=$ $3 \mathcal{A} / 4$, where

$$
\stackrel{\mathrm{o}}{\mathcal{F}}_{\pi \gamma^{*} \gamma^{*}}\left(q_{3}^{2}, q_{4}^{2}\right)=\lim _{m_{q} \rightarrow 0} \mathcal{F}_{\pi \gamma^{*} \gamma^{*}}\left(q_{3}^{2}, q_{4}^{2}\right), F_{0}=\lim _{m_{q} \rightarrow 0} F_{\pi} .
$$

In the dispersive approach of refs. [31, 32, 36, 49], the invariant functions $\mathcal{W}_{i}\left(q_{1}, q_{2}, q_{3}, q_{4}\right)$ are first expressed in terms of a set of appropriate kinematic variables, namely

$$
s=\left(q_{1}+q_{2}\right)^{2}, t=\left(q_{1}+q_{3}\right)^{2}, q_{1}^{2}, q_{2}^{2}, q_{3}^{2}, q_{4}^{2} .
$$

Accordingly, the functions describing the three-point function $\mathcal{W}_{\mu \nu \rho}$ are to be written as $w_{i}\left(q_{3}^{2}, q_{4}^{2}, s\right)$. This rewriting in terms of the variables (3.16) does not change the shortdistance condition (3.1), and the right-hand side involves the same sum, $w_{0}\left(q_{3}^{2}, q_{4}^{2}, s\right)+$ $w_{1}\left(q_{3}^{2}, q_{4}^{2}, s\right)$, as before. The pion-pole contributions in eqs. (3.5) and (3.9) also remain the same, up to the denominators that are now rewritten as $s-M_{\pi}^{2}$. As long as we give the different variables in eq. (3.16) generic values, the whole discussion leading to the condition (3.11) can be repeated again, mutatis mutandis. So let us therefore turn to the kinematic regime relevant for the evaluation of $a_{\mu}^{\mathrm{HLxL}}$. Here the dispersive approach requires to consider the reduced kinematics defined in ref. [36], so that eq. (3.12) is replaced by

$$
\begin{aligned}
\lim _{q_{4} \rightarrow 0} \frac{\partial}{\partial q_{4}^{\sigma}} \mathcal{W}^{\mu \nu \rho \tau}\left(q_{1}, q_{2},-q_{4}-q_{1}-q_{2}, q_{4}\right) \underset{\text { ref. [36] }}{\longrightarrow} & \left.\sum_{i} \mathcal{W}_{i}\left(s, t, q_{1}^{2}, q_{2}^{2}, q_{3}^{2}, q_{4}^{2}\right)\right|_{\substack{t=q_{2}^{2} \\
s=q_{3}^{2} \\
q_{4}^{2}=0}} ^{\substack{q_{2} \\
q_{4}}} \\
& \times \lim _{q_{4} \rightarrow 0} \frac{\partial}{\partial q_{4}^{\sigma}} T_{i}^{\mu \nu \rho \tau}\left(q_{1}, q_{2},-q_{4}-q_{1}-q_{2}, q_{4}\right) .
\end{aligned}
$$

For the contribution from the pion pole to the left-hand side of eq. (3.4) we find (the pion-pole contribution does not depend on the variable $t$ )

$$
\begin{aligned}
\lim _{\substack{s \rightarrow q_{3}^{2} \\
q_{4}^{2} \rightarrow 0}}-\frac{\mathcal{F}_{\pi \gamma^{*} \gamma^{*}}\left((\bar{q}+\hat{q})^{2},(\bar{q}-\hat{q})^{2}\right) \mathcal{F}_{\pi \gamma^{*} \gamma^{*}}\left(q_{3}^{2}, q_{4}^{2}\right)}{s-M_{\pi}^{2}} & =-\frac{\mathcal{F}_{\pi \gamma^{*} \gamma^{*}}\left((\bar{q}+\hat{q})^{2},(\bar{q}-\hat{q})^{2}\right) \mathcal{F}_{\pi \gamma^{*} \gamma^{*}}\left(q_{3}^{2}, 0\right)}{q_{3}^{2}-M_{\pi}^{2}} \\
& =-\frac{2}{3} \frac{F_{\pi}}{\hat{q}^{2}} \frac{\mathcal{F}_{\pi \gamma^{*} \gamma^{*}}\left(q_{3}^{2}, 0\right)}{q_{3}^{2}-M_{\pi}^{2}}+\mathcal{O}\left(\hat{q}^{-4}\right), \quad(3.18)
\end{aligned}
$$

whereas in the same limit its contribution to the right-hand side reads

$$
\lim _{\substack{s \rightarrow q_{3}^{2} \\ q_{4}^{2} \rightarrow 0}}-\frac{2}{\hat{q}^{2}} \operatorname{tr}\left(Q^{2} \lambda^{3}\right) \frac{F_{\pi} \mathcal{F}_{\pi \gamma^{*} \gamma^{*}}\left(q_{3}^{2}, q_{4}^{2}\right)}{s-M_{\pi}^{2}}=-\frac{2}{\hat{q}^{2}} \operatorname{tr}\left(Q^{2} \lambda^{3}\right) \frac{F_{\pi} \mathcal{F}_{\pi \gamma^{*} \gamma^{*}}\left(q_{3}^{2}, 0\right)}{q_{3}^{2}-M_{\pi}^{2}} .
$$

The results for the two sides of the condition (3.4) differ from the previous case, since the second transition form factor now retains a dependence on $q_{3}^{3}$, but what matters is that 
they perfectly match, and this matching persists in the chiral limit, which can be taken without problem.

To summarize this discussion of the pion pole, I find that, in the chiral limit, the short-distance constraint (3.4) leads to

$$
\lim _{q_{4} \rightarrow 0} \lim _{\hat{q}^{2} \rightarrow-\infty} \mathcal{W}_{1}^{\left(\pi^{0}\right)}=-\frac{2}{3} \frac{F_{\pi}}{\hat{q}^{2}} \frac{\mathcal{F}_{\pi \gamma^{*} \gamma^{*}}(0,0)}{q_{3}^{2}}+\mathcal{O}\left(\hat{q}^{-4}\right)=\frac{N_{c}}{18 \pi^{2}} \frac{1}{\hat{q}^{2}} \frac{1}{q_{3}^{2}}+\mathcal{O}\left(\hat{q}^{-4}\right)
$$

in the case where the kinematic configuration corresponding to $q_{4} \rightarrow 0$, and considered by the authors of refs. $[33,34]$, is taken. In the kinematic configuration corresponding to the dispersive treatment of the pion pole advocated by the authors of refs. [31, 32], it instead leads to

$$
\lim _{\substack{s \rightarrow q_{3}^{2} \\ q_{4}^{2} \rightarrow 0}} \lim _{\hat{q}^{2} \rightarrow-\infty} \mathcal{W}_{1}^{\left(\pi^{0}\right)}=-\frac{2}{3} \frac{F_{\pi}}{\hat{q}^{2}} \frac{\mathcal{F}_{\pi \gamma^{*} \gamma^{*}}\left(q_{3}^{2}, 0\right)}{q_{3}^{2}}+\mathcal{O}\left(\hat{q}^{-4}\right)=-\frac{4}{3} \frac{F_{\pi}^{2}}{\hat{q}^{2}}\left(\frac{1}{q_{3}^{2}}\right)^{2}\left[1+\mathcal{O}\left(q_{3}^{-2}\right)\right]+\mathcal{O}\left(\hat{q}^{-4}\right) .
$$

These equations already appear, in some form or other, in refs. [33, 34] as far as (3.20) is concerned, or in refs. [31, 32] in the case of (3.21). The second equality in this last equation holds when $q_{3}^{2}$ becomes large in the Euclidean region (but with $-\hat{q}^{2} \gg-q_{3}^{2}$ ), where the result $[53,54]$

$$
\lim _{q_{3}^{2} \rightarrow-\infty} \mathcal{F}_{\pi \gamma^{*} \gamma^{*}}\left(q_{3}^{2}, 0\right)=\frac{2 F_{\pi}}{q_{3}^{2}}+\mathcal{O}\left(q_{3}^{-4}\right)
$$

can be used. Both limits (3.20) and (3.21) are, as far as I can see, legitimate, in the sense that none reveals any incoherence. However, they will most likely lead to different numerical outcomes as far as the contribution of the pion pole to $a_{\mu}^{\mathrm{HLx}}$ is concerned. But this needs not be a problem per se since what matters in the end is the comparison of the results obtained once all contributions to $a_{\mu}^{\mathrm{HLxL}}$ have been added up.

\subsection{An apparent paradox and its solution}

The debate in the literature on $a_{\mu}^{\mathrm{HLLL}}$ that has resurfaced recently [32, 33] takes its origin in the fact that eq. (3.4) is usually written in terms of the function $w_{L}$,

$\hat{\mathcal{W}}_{1}\left(q_{3}^{2},\left(\bar{q}+\hat{q}+q_{3}\right)^{2},(\bar{q}+\hat{q})^{2},(\bar{q}-\hat{q})^{2}, q_{3}^{2}, 0\right)=-\frac{2}{\hat{q}^{2}} \frac{1}{8 \pi^{2}} w_{L}\left(q_{3}^{2}, 0, q_{3}^{2}\right)+\mathcal{O}\left(\hat{q}^{-4}\right) \quad$ refs. $[32,33]$

As discussed after eq. (2.34), this is quite legitimate in the limit appropriate for the discussion of $a_{\mu}^{\mathrm{HLxL}}$, whether one considers it in the form (3.12) or in the form (3.17). But although $w_{L}\left(q_{3}^{2}, 0, q_{3}^{2}\right) /\left(8 \pi^{2}\right)$ and $w_{0}\left(q_{3}^{2}, 0, q_{3}^{2}\right)+w_{1}\left(q_{3}^{2}, 0, q_{3}^{2}\right)$ are the same functions, they differ by their physical content, and this difference lies at the heart of the debate. In order to explain this point, let me consider the chiral limit and consider the iso-triplet channel, see the first remark after eq. (2.34) for the explanation of the nomenclature and the notation. The discussion in the octet channel is exactly the same, with the $\eta$ meson playing the role of the pion, and extends to the singlet channel and the $\eta^{\prime}$ meson if in addition 
the large- $N_{c}$ limit is taken as well. In the chiral limit, the function $w_{L}^{3}$ is known exactly in QCD, for arbitrary kinematics,

$$
\lim _{m_{q} \rightarrow 0} \frac{1}{8 \pi^{2}} w_{L}^{3}\left(q_{3}^{2}, q_{4}^{2},\left(q_{3}+q_{4}\right)^{2}\right)=\frac{3}{4} \frac{\mathcal{A}}{\left(q_{3}+q_{4}\right)^{2}},
$$

and this single contribution is entirely produced by a dynamical pion pole. Comparing the pion pole in $\hat{\mathcal{W}}_{1}$ with the one in $w_{L}^{3} /\left(8 \pi^{2}\right)$ would lead to compare, in the "dispersive" limit (3.17)

$$
-\frac{2}{3} \frac{F_{0}}{\hat{q}^{2}} \frac{\stackrel{\circ}{\mathcal{F}_{\pi \gamma^{*} \gamma^{*}}\left(q_{3}^{2}, 0\right)}}{q_{3}^{2}}+\mathcal{O}\left(\hat{q}^{-4}\right) \text { vs. }-\frac{2}{3} \frac{F_{0}}{\hat{q}^{2}} \frac{\stackrel{\circ}{\mathcal{F}}_{\pi \gamma^{*} \gamma^{*}}(0,0)}{q_{3}^{2}}+\mathcal{O}\left(\hat{q}^{-4}\right)=-\frac{2}{3} \frac{1}{\hat{q}^{2}} \frac{3}{4} \frac{\mathcal{A}}{q_{3}^{2}}+\mathcal{O}\left(\hat{q}^{-4}\right) .
$$

Clearly, the two expressions cannot match as such for all values of $q_{3}^{2}$, and this mismatch is at the origin of the debate between the authors of refs. [33, 34] on the one hand, and the authors of refs. [31, 32] on the other hand, the former seeing "the dependence on this form factor [i.e. $\left.\stackrel{\circ}{\mathcal{F}}_{\pi \gamma^{*} \gamma^{*}}\left(q_{3}^{2}, 0\right)\right]$ on $q_{3}^{2}$ " as "ambiguous within the dispersive approach", whereas for the latter the model based on a constant form factor [i.e. ${\stackrel{\mathcal{F}}{\pi \gamma^{*} \gamma^{*}}}(0,0)$ in the chiral limit] represents a "distortion" of the low-energy behaviour of the rank-four vacuum polarization tensor. But we have just seen that, although the two ways to implement the kinematic limit relevant for $a_{\mu}^{\mathrm{HLxL}}$ give different results for the pion pole, they are both consistent with the content of eq. (3.4), and the confrontation between the two options in eq. (3.25) never shows up.

In order to understand the origin of this apparent paradox, let us come back to the combination $w_{0}+w_{1}$ that actually appears on the right-hand side of eq. (3.4). Even in the chiral limit, the structure of this function remains quite different from the simple form taken by $w_{L}^{3}$ and given in eq. (3.24),

$$
\begin{aligned}
& \lim _{m_{q} \rightarrow 0}\left[w_{0}^{3}\left(q_{3}^{2}, q_{4}^{2},\left(q_{3}+q_{4}\right)^{2}\right)+w_{1}^{3}\left(q_{3}^{2}, q_{4}^{2},\left(q_{3}+q_{4}\right)^{2}\right)\right]= \\
& =\frac{F_{0} \stackrel{\mathrm{o}}{\mathcal{F}}_{\pi \gamma^{*} \gamma^{*}}\left(q_{3}^{2}, q_{4}^{2}\right)}{\left(q_{3}+q_{4}\right)^{2}}+\Delta w^{3}\left(q_{3}^{2}, q_{4}^{2},\left(q_{3}+q_{4}\right)^{2}\right) \\
& =\frac{3 \mathcal{A} / 4}{\left(q_{3}+q_{4}\right)^{2}}+\frac{F_{0}\left[\mathcal{F}_{\pi \gamma^{*} \gamma^{*}}\left(q_{3}^{2}, q_{4}^{2}\right)-\stackrel{\mathrm{o}}{\mathcal{F}}_{\pi \gamma^{*} \gamma^{*}}(0,0)\right]}{\left(q_{3}+q_{4}\right)^{2}}+\Delta w^{3}\left(q_{3}^{2}, q_{4}^{2},\left(q_{3}+q_{4}\right)^{2}\right) \\
& =\lim _{m_{q} \rightarrow 0} w_{L}^{3}\left(q_{3}^{2}, q_{4}^{2},\left(q_{3}+q_{4}\right)^{2}\right)+\frac{F_{0}\left[\mathcal{\mathcal { F }}_{\pi \gamma^{*} \gamma^{*}}\left(q_{3}^{2}, q_{4}^{2}\right)-\stackrel{\mathrm{o}}{\mathcal{F}}_{\pi \gamma^{*} \gamma^{*}}(0,0)\right]}{\left(q_{3}+q_{4}\right)^{2}}+\Delta w^{3}\left(q_{3}^{2}, q_{4}^{2},\left(q_{3}+q_{4}\right)^{2}\right),
\end{aligned}
$$

where $\Delta w^{3}\left(q_{3}^{2}, q_{4}^{2},\left(q_{3}+q_{4}\right)^{2}\right)$ represents the part of $w_{0}+w_{1}$ that is regular at $\left(q_{3}+q_{4}\right)^{2}=0$ in the chiral limit. The first equality gives the version of eq. (3.9) corresponding to the chiral limit. In the second equality I have isolated the contribution to the pole coming from $\stackrel{\circ}{\mathcal{F}}_{\pi \gamma^{*} \gamma^{*}}(0,0)$ alone, and have identified it, in the third equality, with eq. (3.24). Taking now the limit where $q_{4}$ vanishes or, equivalently, the combined limit $q_{4}^{2} \rightarrow 0$ and $\left(q_{3}+q_{4}\right)^{2} \rightarrow q_{3}^{2}$, 
we see that the relation

$$
\begin{aligned}
\lim _{q_{4} \rightarrow 0} \lim _{m_{q} \rightarrow 0}\left[w_{0}^{3}\left(q_{3}^{2}, q_{4}^{2},\left(q_{3}+q_{4}\right)^{2}\right)+w_{1}^{3}\left(q_{3}^{2}, q_{4}^{2},\left(q_{3}+q_{4}\right)^{2}\right)\right] & =\lim _{q_{4} \rightarrow 0} \lim _{m_{q} \rightarrow 0} w_{L}^{3}\left(q_{3}^{2}, q_{4}^{2},\left(q_{3}+q_{4}\right)^{2}\right) \\
& =\frac{3 \mathcal{A} / 4}{q_{3}^{2}}
\end{aligned}
$$

which follows from eq. (2.35), rests on an exact cancellation between a contribution that comes from a part of the pion pole, namely the one involving the momentum dependence of the pion transition form factor, and the contribution that is regular at $\left(q_{3}+q_{4}\right)^{2}=0$,

$$
\frac{F_{0}\left[\stackrel{\circ}{\mathcal{F}}_{\pi \gamma^{*} \gamma^{*}}\left(q_{3}^{2}, 0\right)-\stackrel{\circ}{\mathcal{F}}_{\pi \gamma^{*} \gamma^{*}}(0,0)\right]}{q_{3}^{2}}+\Delta w^{3}\left(q_{3}^{2}, 0, q_{3}^{2}\right)=0 .
$$

The computation in appendix B shows that this cancellation indeed happens at one loop in the low-energy expansion. But it is in fact an exact property of QCD in the chiral limit, and a direct consequence of the relation (2.34). Besides its confirmation in the low-energy expansion, it can also be illustrated in a simple resonance model like the one of ref. [55]. A straightforward calculation yields

$$
\begin{aligned}
w_{0}^{3}\left(q_{3}^{2}, q_{4}^{2},\left(q_{3}+q_{4}\right)^{2}\right)+w_{1}^{3}\left(q_{3}^{2}, q_{4}^{2},\left(q_{3}+q_{4}\right)^{2}\right)= & \frac{1}{\left(q_{3}+q_{4}\right)^{2}}\left[\frac{3}{4} \mathcal{A}+\frac{b\left(q_{3}^{2}+q_{4}^{2}\right)}{\left(q_{3}^{2}-M_{V}^{2}\right)\left(q_{4}^{2}-M_{V}^{2}\right)}\right. \\
& \left.+\frac{c_{1} q_{3}^{2}+c_{2} q_{4}^{2}}{q_{3}^{2}-M_{V}^{2}}+\frac{c_{1} q_{4}^{2}+c_{2} q_{3}^{2}}{q_{4}^{2}-M_{V}^{2}}\right] \\
& -\frac{b}{\left(q_{3}^{2}-M_{V}^{2}\right)\left(q_{4}^{2}-M_{V}^{2}\right)}-\frac{c_{1}}{q_{3}^{2}-M_{V}^{2}}-\frac{c_{2}}{q_{4}^{2}-M_{V}^{2}} .
\end{aligned}
$$

The manner in which the parameters $b, c_{1}, c_{2}$ are related to the resonance couplings and to the mass $M_{V}$ of the vector resonance in this model need not concern us here. What matters instead is to observe that the cancellation (3.28) indeed takes place when either one of the limit (3.12) or (3.17) is considered.

Whatever one decides to call the pseudo-paradox (3.25) at the origin of the debate in the recent literature, it rests on a wrong identification, in the chiral limit, of the pion-pole contribution on the right-hand side of the short-distance constraint in eq. (3.4), and which itself arises from the identification of the two functions $w_{L}$ and $w_{0}+w_{1}$ in the kinematic limit relevant for $a_{\mu}^{\mathrm{HLxL}}$. This second identification is correct from the functional point of view, but the quite different physical contents of these two functions have not been given sufficiently close attention. Once this is done, the debate loses its raison d'être.

\subsection{Pseudoscalar poles}

We may now extend the discussion to pseudoscalar poles in general. Strictly speaking, poles appear only for the lightest of these states, the pseudo-Goldstone mesons $\pi^{0}, \eta, \eta^{\prime}$. Heavier pseudoscalar states, like for instance the $\pi(1300)$ iso-triplet $J^{P}=0^{-}$resonance, are often too broad to be described just as poles on the real axis of the complex $s$-plane. Such a description would require a narrow-width approximation, which finds some justification by considering, for instance, the large- $N_{c}$ limit. Let us adopt the latter framework for the 
present discussion. In the case of the three-point function $\mathcal{W}_{\mu \nu \rho}$, these poles are again to be found in the function $w_{0}$

$$
w_{0}\left(q_{3}^{2}, q_{4}^{2},\left(q_{3}+q_{4}\right)^{2}\right)=\sum_{P} \sum_{a=3,8,0} \operatorname{tr}\left(Q^{2} \lambda^{a}\right) \frac{F_{P}^{a} \mathcal{F}_{P \gamma^{*} \gamma^{*}}\left(q_{3}^{2}, q_{4}^{2}\right)}{\left(q_{3}+q_{4}\right)^{2}-M_{P}^{2}}+\cdots .
$$

Here the sum runs over all the $J^{P}=0^{-}$states with masses $M_{P}$, decay constants $F_{P}^{a}$, defined by the matrix elements

$$
\left\langle\Omega\left|A_{\rho}^{a}(0)\right| P(p)\right\rangle=i F_{P}^{a} p_{\rho},
$$

and with transitions form factors $\mathcal{F}_{P \gamma^{*} \gamma^{*}}$ defined in analogy with the case of the pion in eq. (3.6).

At the level of the four-point function, each of these pseudoscalar states produces a contribution analogous to the one of the pion,

$$
\hat{\mathcal{W}}_{1}^{(P)}\left(q_{1}, q_{2}, q_{3}, q_{4}\right)=-\frac{\mathcal{F}_{P \gamma^{*} \gamma^{*}}\left(q_{1}^{2}, q_{2}^{2}\right) \mathcal{F}_{P \gamma^{*} \gamma^{*}}\left(q_{3}^{2}, q_{4}^{2}\right)}{\left(q_{3}+q_{4}\right)^{2}-M_{P}^{2}} .
$$

Since the dynamical singularities have to match on both sides of the short-distance constraint (3.4), we need to check that the relation

$$
\hat{\mathcal{W}}_{1}^{(P)}\left(\bar{q}+\hat{q}, \bar{q}-\hat{q}, q_{3}, q_{4}\right)=-\frac{2}{\hat{q}^{2}} \sum_{a=3,8,0} \operatorname{tr}\left(Q^{2} \lambda^{a}\right) \frac{F_{P}^{a} \mathcal{F}_{P \gamma^{*} \gamma^{*}}\left(q_{3}^{2}, q_{4}^{2}\right)}{\left(q_{3}+q_{4}\right)^{2}-M_{P}^{2}}+\mathcal{O}\left(\hat{q}^{-4}\right)
$$

holds for asymptotic Euclidean values of the momentum $\hat{q}$. That this is indeed the case follows again from eq. (2.9). It is thus possible to consider the two limits discussed previously for the pion-pole contribution to $a_{\mu}^{\mathrm{HLxL}}$. Without surprise, the outcomes are again different

$$
\begin{aligned}
\lim _{q_{4} \rightarrow 0} \lim _{\hat{q}^{2} \rightarrow-\infty} \hat{\mathcal{W}}_{1}^{(P)}\left(\bar{q}+\hat{q}, \bar{q}-\hat{q}, q_{3}, q_{4}\right)= & -\frac{2}{\hat{q}^{2}} \sum_{a=3,8,0} \operatorname{tr}\left(Q^{2} \lambda^{a}\right) \frac{F_{P}^{a} \mathcal{F}_{P \gamma^{*} \gamma^{*}}\left(M_{P}^{2}, 0\right)}{q_{3}^{2}-M_{P}^{2}} \\
& +\mathcal{O}\left(\hat{q}^{-4}\right) \\
\lim _{\substack{s \rightarrow q_{3}^{2} \\
\hat{q}_{4}^{2} \rightarrow 0}} \lim _{\substack{2 \\
q^{2} \rightarrow-\infty}} \hat{\mathcal{W}}_{1}^{(P)}\left(\bar{q}+\hat{q}, \bar{q}-\hat{q}, q_{3}, q_{4}\right)= & -\frac{2}{\hat{q}^{2}} \sum_{a=3,8,0} \operatorname{tr}\left(Q^{2} \lambda^{a}\right) \frac{F_{P}^{a} \mathcal{F}_{P \gamma^{*} \gamma^{*}}\left(q_{3}^{2}, 0\right)}{q_{3}^{2}-M_{P}^{2}} \\
& +\mathcal{O}\left(\hat{q}^{-4}\right)
\end{aligned}
$$

Finally, in the combined large- $N_{c}$ and three-flavour chiral limit, each one of the flavourdiagonal axial currents defined in eq. (2.10) is conserved, so that the decay constants vanish as $F_{P}^{a} \sim \mathcal{O}\left(m_{q}\right)+\mathcal{O}\left(1 / N_{c}\right)$ for $P \neq \pi^{0}, \eta, \eta^{\prime}$, and the non-Goldstone pseudoscalar poles in $\hat{\mathcal{W}}_{1}$ contribute only to sub-leading terms of the short-distance expansion.

\section{Summary and conclusion}

This note proposes a critical, albeit only partial, discussion of the implications of the shortdistance constraint of ref. [34] for one of the invariant functions describing the rank-four 
hadronic vacuum polarization tensor. This study is focused on a very specific issue under debate in the recent literature, with the hope that it may contribute positively to this discussion. To this effect, I have first re-derived the short-distance constraints in the more general case of a generic kinematic configuration, and, more importantly, expressed them in terms of functions that are free of kinematic singularities. This allows to state a certain number of general properties that have to be met and that have been listed after eq. (3.4).

I have then discussed the two kinematic limits that are currently considered in applications to the anomalous magnetic moment of the muon, for both the contribution from the pion pole or from narrow non-Goldstone pseudoscalar states. Working with functions that are free of kinematic singularities warrants that both kinematic limits can be taken without problem or ambiguity, and lead to coherent results if the same limit is taken on both sides of eq. (3.4). They are, however, definitely different limits, and as such simply give... different results for the contribution from these poles. In itself, this needs not necessarily constitute a problem, since the pion pole is but one contribution to $a_{\mu}^{\mathrm{HLx}}$, although an important one. But an evaluation of $a_{\mu}^{\mathrm{HLx}}$ at a level of precision of $10 \%$ in relative terms requires also to include other contributions in a controlled manner, and a comparison between different approaches or prescriptions is only meaningful once this task has been completed.

If one follows the evolution of the pion pole through the different limits that are taken, no ambiguity in its identification arises. But in order to bring the two functions $w_{L}\left(q_{3}^{2}, 0, q_{3}^{2}\right)$ and $w_{0}\left(q_{3}^{2}, 0, q_{3}^{2}\right)+w_{1}\left(q_{3}^{2}, 0, q_{3}^{2}\right)$ to an identical form, a cancellation mechanism, between part of the pion pole contribution to $w_{0}\left(q_{3}^{2}, 0, q_{3}^{2}\right)+w_{1}\left(q_{3}^{2}, 0, q_{3}^{2}\right)$ and the contributions without pion pole, necessarily needs to be at work in QCD. This cancellation mechanism, which is brought out in eq. (3.28) and is clearly evidenced in the regime of small momentum transfers, where the low-energy expansion can be used, constitutes the major new result of this study. The function that appears on the right-hand side of the short-distance constraint on $\hat{\mathcal{W}}_{1}\left(q_{1}, q_{2}, q_{3}, q_{4}\right)$ is actually $w_{0}\left(q_{3}^{2}, 0, q_{3}^{2}\right)+w_{1}\left(q_{3}^{2}, 0, q_{3}^{2}\right)$, whose pion-pole contribution in the chiral limit is thus only partially reproduced by the pion pole of $w_{L}\left(q_{3}^{2}, 0, q_{3}^{2}\right)$.

Narrow pseudoscalar states other than $\pi, \eta, \eta^{\prime}$ contribute to both sides of eq. (3.4) in a perfectly consistent manner. And this consistency persists in the chiral limit, where the non-singlet and non-Goldstone pseudoscalar states disappear altogether from the righthand side while their contribution to the left-hand side becomes sub-leading in the shortdistance expansion. If one takes in addition the large- $N_{c}$ limit, then this situation extends to all non-Goldstone pseudoscalar states.

Finally, let me point out that although I have referred several times to the constraint (3.4) or to its more general version (2.12) as the short-distance condition, the plural form would actually be more appropriate, since it really is a constraint on the fourth rank vacuum polarization tensor for each value of the momentum transfers $q_{3}^{2}, q_{4}^{2}$ and $\left(q_{3}+q_{4}\right)^{2}$. And even in the kinematic regime relevant for the evaluation of $a_{\mu}^{\mathrm{HLxL}}$ it still gives a condition for each value of $q_{3}^{2}$ and not only when $q_{3}^{2}$ becomes large in the Euclidean region, as it is most of the time being used. No phenomenological approach or model designed for the evaluation of $a_{\mu}^{\mathrm{HLLL}} \mathrm{I}$ am aware of has, so far, exploited the full content of the condition of ref. [34] in this broader sense. 
Note added. After the preprint version of this article had been made public on arXiv, the preprint [56] appeared, where the authors address related issues.

\section{Acknowledgments}

I wish to thank E. de Rafael for a careful reading of the manuscript, for useful suggestions, and for many stimulating and informative discussions about g-2 related issues over the years.

\section{A Technical aspects of the short-distance expansion}

In this appendix, I describe how the short-distance constraint given in eq. (3.1) for the four-point function $\mathcal{W}^{\mu \nu \rho \sigma}$ can be transformed into short-distance constraints for some of the individual invariant functions $\mathcal{W}_{i}$ introduced in eq. (2.8). This is a somewhat lengthy process, so that only a brief outline of it will be presented. Before that, I first give the dictionary between the notation used here and the one used in refs. [31, 32, 36, 49].

The tensor $\Pi^{\mu \nu \rho \sigma}\left(q_{1}, q_{2}, q_{3}, q_{4}\right)$ defined in eq. (3.1) of [36] is related to $\mathcal{W}^{\mu \nu \rho \sigma}\left(q_{1}, q_{2}, q_{3}, q_{4}\right)$ by

$$
\mathcal{W}^{\mu \nu \rho \sigma}\left(q_{1}, q_{2}, q_{3}, q_{4}\right)=\Pi^{\mu \nu \rho \sigma}\left(-q_{1},-q_{2},-q_{3}, q_{4}\right)=\Pi^{\mu \nu \rho \sigma}\left(q_{1}, q_{2}, q_{3},-q_{4}\right) .
$$

The absence of a minus sign in the last entry of $\Pi^{\mu \nu \rho \sigma}$ in the first equality is due to the fact that in refs. $[36,49]$ the momenta $q_{1}, q_{2}$ and $q_{3}$ are taken as incoming, whereas $q_{4}$ is taken as outgoing. The second equality follows from the fact that the tensors $\Pi^{\mu \nu \rho \sigma}\left(q_{1}, q_{2}, q_{3}, q_{4}\right)$ or $\mathcal{W}^{\mu \nu \rho \sigma}\left(q_{1}, q_{2}, q_{3}, q_{4}\right)$ remain the same if all momenta are reversed simultaneously. The tensors $T_{i}^{\mu \nu \rho \sigma}$ are listed in eq. (3.14) and in appendix B of ref. [36]. I have taken the "seed tensors" displayed in eq. (3.14) of ref. [36] as they stand, i.e. without changing the sign of $q_{4}$, and have then applied the symmetry operations listed in eq. (B.1) of ref. [36], with the difference that the exchange operation $\mathcal{C}_{14}$, for instance, means $\left(\mu, q_{1}\right) \leftrightarrow\left(\sigma, q_{4}\right)$, i.e. without changing the sign of $q_{4}$. The relations between the invariant functions then read $\Pi_{i}\left(q_{1}, q_{2}, q_{3}, q_{4}\right)= \pm \mathcal{W}_{i}\left(q_{1}, q_{2}, q_{3}, q_{4}\right)$ and it is easy to trace which sign applies for a specific value of $i$. This explains, for instance, why in the definition of $\hat{W}_{1}$ in eq. (3.3) there is a relative minus sign between the two terms, whereas one finds a plus sign in eq. (2.15) of ref. [49].

Coming back to eq. (3.1), each function $\mathcal{W}_{i}$ has, in the limit under consideration, an expansion of the form

$$
\mathcal{W}_{i}\left(\bar{q}+\hat{q}, \bar{q}-\hat{q}, q_{3}, q_{4}\right)=\frac{1}{\hat{q}^{n_{i}}}\left[\mathcal{W}_{i}^{\left[n_{i}\right]}\left(q_{3}, q_{4}\right)+\frac{\hat{q}^{\mu}}{\hat{q}^{2}} \mathcal{W}_{i, \mu}^{\left[n_{i}+1\right]}\left(q_{3}, q_{4}\right)+\cdots\right] .
$$

The value of $n_{i}$, which determines the leading power behaviour, can be fixed in the following manner: the tensors $T_{i}^{\mu \nu \rho \sigma}$ have dimension 4 for $i=1, \ldots, 6$, dimension 8 for $i=31, \ldots, 36$, and dimension 6 in all other cases, whereas the tensor $\mathcal{W}^{\mu \nu \rho \sigma}$ is dimensionless. Furthermore, we are looking for relations of the type

$$
\mathcal{W}_{i}^{\left[n_{i}\right]}\left(q_{3}, q_{4}\right)=\sum_{k=0}^{3} c_{i k} w_{k}\left(q_{3}, q_{4}\right),
$$


with some numerical coefficients $c_{i k}$, and where the functions $w_{k}\left(q_{3}, q_{4}\right)$ have dimension -2 . This means that one has $n_{i}=2$ for $i=1, \ldots, 6, n_{i}=6$ for $i=31, \ldots, 36$, and $n_{i}=4$ for the remaining values of $i$. It is then possible to proceed upon going through the following steps:

- First, one notices that the highest power in $\hat{q}$ of each tensor $T_{i}^{\mu \nu \rho \sigma}\left(\bar{q}+\hat{q}, \bar{q}-\hat{q}, q_{3}, q_{4}\right)$, which is given by

$$
\widehat{T}_{i}^{\mu \nu \rho \sigma}\left(\hat{q}, q_{3}, q_{4}\right) \equiv T_{i}^{\mu \nu \rho \sigma}\left(\hat{q},-\hat{q}, q_{3}, q_{4}\right),
$$

also varies from case to case. This highest power is simply equal to 1 for $i=1$, to 2 for $i=2, \ldots, 6,9,12,13,15,17,18,29,30,32,37,48,49$, and so on. Since we are looking for a behaviour that does not decrease faster than $1 / \hat{q}$ when $\hat{q}$ becomes large, we are eventually left with only the cases

$$
i=1, \ldots, 8,10,11,14,16,19, \ldots, 28,31,38, \ldots 47,50, \ldots 54
$$

to consider.

- Second, for each of these cases, one extracts from the tensor $T_{i}^{\mu \nu \rho \sigma}\left(\bar{q}+\hat{q}, \bar{q}-\hat{q}, q_{3}, q_{4}\right)$ the part, denoted as $\mathcal{T}_{i}^{\mu \nu \rho \sigma}\left(\hat{q}, q_{3}, q_{4}\right)$, that is either linear in $\hat{q}$, i.e.

$$
\mathcal{T}_{i}^{\mu \nu \rho \sigma}\left(\hat{q}, q_{3}, q_{4}\right)=T_{i}^{\mu \nu \rho \sigma}\left(\hat{q}, \bar{q}, q_{3}, q_{4}\right)+T_{i}^{\mu \nu \rho \sigma}\left(\bar{q},-\hat{q}, q_{3}, q_{4}\right),
$$

for $i=2, \ldots, 6$, or that is of the form $\hat{q}^{2}$ times terms linear in $\hat{q}$, when it exists, in the other cases, except $i=1$, where one has

$$
\mathcal{T}_{1}^{\mu \nu \rho \sigma}\left(\hat{q}, q_{3}, q_{4}\right)=T_{1}^{\mu \nu \rho \sigma}\left(\bar{q}+\hat{q}, \bar{q}-\hat{q}, q_{3}, q_{4}\right)=K_{0}^{\mu \nu \rho \sigma}\left(\hat{q}, q_{3}, q_{4}\right) .
$$

With these pieces at hand, one can then construct a set of other useful relations involving the tensors $K_{i}^{\mu \nu \rho \sigma}\left(\hat{q}, q_{3}, q_{4}\right)$ defined in eq. (3.2):

$$
\begin{aligned}
K_{0}^{\mu \nu \rho \sigma}\left(\hat{q}, q_{3}, q_{4}\right)= & \frac{1}{\hat{q}^{2}}\left[\mathcal{T}_{46}^{\mu \nu \rho \sigma}\left(\hat{q}, q_{3}, q_{4}\right)+\mathcal{T}_{47}^{\mu \nu \rho \sigma}\left(\hat{q}, q_{3}, q_{4}\right)\right] \\
& -2\left[\mathcal{T}_{5}^{\mu \nu \rho \sigma}\left(\hat{q}, q_{3}, q_{4}\right)+\mathcal{T}_{6}^{\mu \nu \rho \sigma}\left(\hat{q}, q_{3}, q_{4}\right)\right] \\
K_{3}^{\mu \nu \rho \sigma}\left(\hat{q}, q_{3}, q_{4}\right) & \begin{aligned}
& \frac{1}{2} K_{1}^{\mu \nu \rho \sigma}\left(\hat{q}, q_{3}, q_{4}\right)= \frac{1}{\hat{q}^{2}}\left[x_{1} \mathcal{T}_{10}^{\mu \nu \rho \sigma}\left(\hat{q}, q_{3}, q_{4}\right)-y_{1} \mathcal{T}_{11}^{\mu \nu \rho \sigma}\left(\hat{q}, q_{3}, q_{4}\right)\right. \\
&\left.+\left(1-y_{1}\right) \mathcal{T}_{14}^{\mu \nu \rho \sigma}\left(\hat{q}, q_{3}, q_{4}\right)-\left(1-x_{1}\right) \mathcal{T}_{16}^{\mu \nu \rho \sigma}\left(\hat{q}, q_{3}, q_{4}\right)\right] \\
&+\frac{1}{2 \hat{q}^{2}}\left[\mathcal{T}_{46}^{\mu \nu \rho \sigma}\left(\hat{q}, q_{3}, q_{4}\right)-\mathcal{T}_{47}^{\mu \nu \rho \sigma}\left(\hat{q}, q_{3}, q_{4}\right)\right] \\
& K_{2}^{\mu \nu \rho \sigma}\left(\hat{q}, q_{3}, q_{4}\right)= \frac{x_{2}}{\hat{q}^{2}}\left[\mathcal{T}_{50}^{\mu \nu \rho \sigma}\left(\hat{q}, q_{3}, q_{4}\right)+\mathcal{T}_{51}^{\mu \nu \rho \sigma}\left(\hat{q}, q_{3}, q_{4}\right)\right. \\
&+\frac{\left.1-\mathcal{T}_{52}^{\mu \nu \rho \sigma}\left(\hat{q}, q_{3}, q_{4}\right)+\mathcal{T}_{53}^{\mu \nu \rho \sigma}\left(\hat{q}, q_{3}, q_{4}\right)\right]}{\hat{q}^{2}}\left[y_{2}\left(\mathcal{T}_{38}^{\mu \nu \rho \sigma}\left(\hat{q}, q_{3}, q_{4}\right)+\mathcal{T}_{39}^{\mu \nu \rho \sigma}\left(\hat{q}, q_{3}, q_{4}\right)\right)\right. \\
&+\left(1-y_{2}\right)\left(\mathcal{T}_{40}^{\mu \nu \rho \sigma}\left(\hat{q}, q_{3}, q_{4}\right)+\mathcal{T}_{41}^{\mu \nu \rho \sigma}\left(\hat{q}, q_{3}, q_{4}\right)\right) \\
&-\mathcal{T}_{21}^{\mu \nu \rho \sigma}\left(\hat{q}, q_{3}, q_{4}\right)-\mathcal{T}_{23}^{\mu \nu \rho \sigma}\left(\hat{q}, q_{3}, q_{4}\right) \\
&\left.-\mathcal{T}_{25}^{\mu \nu \rho \sigma}\left(\hat{q}, q_{3}, q_{4}\right)-\mathcal{T}_{27}^{\mu \nu \rho \sigma}\left(\hat{q}, q_{3}, q_{4}\right)\right]
\end{aligned} \\
&
\end{aligned}
$$




$$
\begin{aligned}
K_{3}^{\mu \nu \rho \sigma}\left(\hat{q}, q_{3}, q_{4}\right)=\frac{x_{3}}{\hat{q}^{2}}\left[\mathcal{T}_{50}^{\mu \nu \rho \sigma}\left(\hat{q}, q_{3}, q_{4}\right)+\mathcal{T}_{51}^{\mu \nu \rho \sigma}\left(\hat{q}, q_{3}, q_{4}\right)\right. \\
\left.-\mathcal{T}_{52}^{\mu \nu \rho \sigma}\left(\hat{q}, q_{3}, q_{4}\right)-\mathcal{T}_{53}^{\mu \nu \rho \sigma}\left(\hat{q}, q_{3}, q_{4}\right)\right] \\
-\frac{1-x_{3}}{\hat{q}^{2}}\left[y_{3}\left(\mathcal{T}_{38}^{\mu \nu \rho \sigma}\left(\hat{q}, q_{3}, q_{4}\right)-\mathcal{T}_{39}^{\mu \nu \rho \sigma}\left(\hat{q}, q_{3}, q_{4}\right)\right)\right. \\
-\left(1-y_{3}\right)\left(\mathcal{T}_{40}^{\mu \nu \rho \sigma}\left(\hat{q}, q_{3}, q_{4}\right)-\mathcal{T}_{41}^{\mu \nu \rho \sigma}\left(\hat{q}, q_{3}, q_{4}\right)\right) \\
+\mathcal{T}_{21}^{\mu \nu \rho \sigma}\left(\hat{q}, q_{3}, q_{4}\right)-\mathcal{T}_{23}^{\mu \nu \rho \sigma}\left(\hat{q}, q_{3}, q_{4}\right) \\
\left.+\mathcal{T}_{25}^{\mu \nu \rho \sigma}\left(\hat{q}, q_{3}, q_{4}\right)-\mathcal{T}_{27}^{\mu \nu \rho \sigma}\left(\hat{q}, q_{3}, q_{4}\right)\right]
\end{aligned}
$$

In these identities $x_{1,2,3}$ and $y_{1,2,3}$ are real parameters belonging to the interval $[0,1]$, but can otherwise be chosen arbitrarily. To these, one also has to add the two following relations:

$$
\begin{aligned}
\frac{\hat{q}^{2}}{2}\left[K_{2}^{\mu \nu \rho \sigma}\left(\hat{q}, q_{3}, q_{4}\right)+K_{3}^{\mu \nu \rho \sigma}\left(\hat{q}, q_{3}, q_{4}\right)\right]= & \widehat{T}_{42}^{\mu \nu \rho \sigma}\left(\hat{q}, q_{3}, q_{4}\right)+\widehat{T}_{43}^{\mu \nu \rho \sigma}\left(\hat{q}, q_{3}, q_{4}\right) \\
+\left(\hat{q} \cdot q_{3}\right) & {\left[\widehat{T}_{2}^{\mu \nu \rho \sigma}\left(\hat{q}, q_{3}, q_{4}\right)-\widehat{T}_{3}^{\mu \nu \rho \sigma}\left(\hat{q}, q_{3}, q_{4}\right)\right.} \\
& \left.-\widehat{T}_{5}^{\mu \nu \rho \sigma}\left(\hat{q}, q_{3}, q_{4}\right)+\widehat{T}_{6}^{\mu \nu \rho \sigma}\left(\hat{q}, q_{3}, q_{4}\right)\right],
\end{aligned}
$$

and

$$
\begin{aligned}
\frac{\hat{q}^{2}}{2}\left[K_{2}^{\mu \nu \rho \sigma}\left(\hat{q}, q_{3}, q_{4}\right)-K_{3}^{\mu \nu \rho \sigma}\left(\hat{q}, q_{3}, q_{4}\right)\right]= & \widehat{T}_{44}^{\mu \nu \rho \sigma}\left(\hat{q}, q_{3}, q_{4}\right)+\widehat{T}_{45}^{\mu \nu \rho \sigma}\left(\hat{q}, q_{3}, q_{4}\right) \\
-\left(\hat{q} \cdot q_{4}\right) & {\left[\widehat{T}_{2}^{\mu \nu \rho \sigma}\left(\hat{q}, q_{3}, q_{4}\right)-\widehat{T}_{3}^{\mu \nu \rho \sigma}\left(\hat{q}, q_{3}, q_{4}\right)\right.} \\
& \left.-\widehat{T}_{5}^{\mu \nu \rho \sigma}\left(\hat{q}, q_{3}, q_{4}\right)+\widehat{T}_{6}^{\mu \nu \rho \sigma}\left(\hat{q}, q_{3}, q_{4}\right)\right] .
\end{aligned}
$$

- Next, one expands the functions $\mathcal{W}_{i}$ as explained in eq. (A.2), taking into account the symmetry properties of these functions that are listed in ref. [36]. It then remains to collect in the four-point function all the terms that do not decrease faster than $1 / \hat{q}$ and to require that their sum matches the right-hand side of eq. (3.1). The result of this exercise then leads to the following relations:

$$
\begin{aligned}
& \mathcal{W}_{1}^{[2]}\left(q_{3}, q_{4}\right)+\frac{1}{2}[\left.\mathcal{W}_{46}^{[4]}\left(q_{3}, q_{4}\right)+\mathcal{W}_{46}^{[4]}\left(q_{4}, q_{3}\right)\right]=-2 w_{0}\left(q_{3}, q_{4}\right), \\
& \frac{1}{2}\left[\mathcal{W}_{46}^{[4]}\left(q_{3}, q_{4}\right)-\mathcal{W}_{46}^{[4]}\left(q_{4}, q_{3}\right)\right]=+2 w_{1}\left(q_{3}, q_{4}\right), \\
& {\left[\mathcal{W}_{38}^{[4]}\left(q_{3}, q_{4}\right)+\mathcal{W}_{38}^{[4]}\left(q_{4}, q_{3}\right)\right] } \\
&+\frac{1}{2}\left[\mathcal{W}_{42}^{[4]}\left(q_{3}, q_{4}\right)+\mathcal{W}_{42}^{[4]}\left(q_{4}, q_{3}\right)+\mathcal{W}_{50}^{[4]}\left(q_{3}, q_{4}\right)+\mathcal{W}_{50}^{[4]}\left(q_{4}, q_{3}\right)\right]=-2 w_{2}\left(q_{3}, q_{4}\right), \\
& {\left[\mathcal{W}_{46}^{[4]}\left(q_{3}, q_{4}\right)-\mathcal{W}_{46}^{[4]}\left(q_{4}, q_{3}\right)\right]-\left[\mathcal{W}_{38}^{[4]}\left(q_{3}, q_{4}\right)-\mathcal{W}_{38}^{[4]}\left(q_{4}, q_{3}\right)\right] } \\
&+\frac{1}{2}\left[\mathcal{W}_{42}^{[4]}\left(q_{3}, q_{4}\right)-\mathcal{W}_{42}^{[4]}\left(q_{4}, q_{3}\right)+\mathcal{W}_{50}^{[4]}\left(q_{3}, q_{4}\right)-\mathcal{W}_{50}^{[4]}\left(q_{4}, q_{3}\right)\right]=-2 w_{3}\left(q_{3}, q_{4}\right),
\end{aligned}
$$


together with

$$
\begin{aligned}
\mathcal{W}_{2}^{[2]}\left(q_{3}, q_{4}\right)=\mathcal{W}_{3}^{[2]}\left(q_{3}, q_{4}\right)=\mathcal{W}_{4}^{[2]}\left(q_{3}, q_{4}\right) & =0, \\
\mathcal{W}_{2, \mu}^{[3]}\left(q_{3}, q_{4}\right)-q_{3 \mu} \mathcal{W}_{42}^{[4]}\left(q_{3}, q_{4}\right)+q_{4 \mu} \mathcal{W}_{42}^{[4]}\left(q_{4}, q_{3}\right) & =0, \\
\mathcal{W}_{5}^{[2]}\left(q_{3}, q_{4}\right)+\mathcal{W}_{10}^{[4]}\left(q_{3}, q_{4}\right)+\mathcal{W}_{10}^{[4]}\left(q_{4}, q_{3}\right) & =0, \\
\mathcal{W}_{10}^{[4]}\left(q_{3}, q_{4}\right) & =\mathcal{W}_{46}^{[4]}\left(q_{3}, q_{4}\right), \\
\mathcal{W}_{5, \mu}^{[3]}\left(q_{3}, q_{4}\right)+\mathcal{W}_{10, \mu}^{[5]}\left(q_{3}, q_{4}\right)-\mathcal{W}_{10, \mu}^{[5]}\left(q_{4}, q_{3}\right) & \\
+q_{3 \mu}\left[\mathcal{W}_{22}^{[4]}\left(q_{3}, q_{4}\right)+\mathcal{W}_{42}^{[4]}\left(q_{3}, q_{4}\right)\right] & \\
-q_{4 \mu}\left[\mathcal{W}_{22}^{[4]}\left(q_{4}, q_{3}\right)+\mathcal{W}_{42}^{[4]}\left(q_{4}, q_{3}\right)\right]-\mathcal{W}_{54, \mu}^{[5]}\left(q_{3}, q_{4}\right) & =0, \\
\mathcal{W}_{7}^{[4]}\left(q_{3}, q_{4}\right) & =\mathcal{W}_{19}^{[4]}\left(q_{3}, q_{4}\right), \\
\mathcal{W}_{21}^{[4]}\left(q_{3}, q_{4}\right) & =-2 \mathcal{W}_{38}^{[4]}\left(q_{4}, q_{3}\right) .
\end{aligned}
$$

Introducing, along eqs. (2.15) and (2.16) of ref. [49], the functions $\hat{\mathcal{W}}_{i}$, with the appropriate changes of signs due to the differences in the conventions, as discussed after eq. (A.1) above, one then establishes eq. (3.4) and, for instance,

$$
\begin{aligned}
& \hat{\mathcal{W}}_{5}\left(\bar{q}+\hat{q}, \bar{q}-\hat{q}, q_{3}, q_{4}\right)+\hat{\mathcal{W}}_{6}\left(\bar{q}+\hat{q}, \bar{q}-\hat{q}, q_{3}, q_{4}\right) \\
& \begin{array}{l}
-\hat{q}^{2}\left[\hat{\mathcal{W}}_{50}\left(\bar{q}+\hat{q}, \bar{q}-\hat{q}, q_{3}, q_{4}\right)+\hat{\mathcal{W}}_{51}\left(\bar{q}+\hat{q}, \bar{q}-\hat{q}, q_{3}, q_{4}\right)\right] \\
\quad=\frac{4}{\hat{q}^{2}}\left[w_{2}\left(q_{3}^{2}, q_{4}^{2},\left(q_{3}+q_{4}\right)^{2}\right)+w_{3}\left(q_{3}^{2}, q_{4}^{2},\left(q_{3}+q_{4}\right)^{2}\right)\right]+\mathcal{O}\left(\hat{q}^{-4}\right),
\end{array}
\end{aligned}
$$

or

$$
\begin{gathered}
\hat{\mathcal{W}}_{50}\left(\bar{q}+\hat{q}, \bar{q}-\hat{q}, q_{3}, q_{4}\right)+\hat{\mathcal{W}}_{51}\left(\bar{q}+\hat{q}, \bar{q}-\hat{q}, q_{3}, q_{4}\right)+2 \hat{\mathcal{W}}_{39}\left(\bar{q}+\hat{q}, \bar{q}-\hat{q}, q_{3}, q_{4}\right)=(\mathrm{A} .21) \\
=-\frac{4}{\hat{q}^{4}}\left[w_{2}\left(q_{3}^{2}, q_{4}^{2},\left(q_{3}+q_{4}\right)^{2}\right)+w_{3}\left(q_{3}^{2}, q_{4}^{2},\left(q_{3}+q_{4}\right)^{2}\right)\right]+\mathcal{O}\left(\hat{q}^{-6}\right) .
\end{gathered}
$$

The limit $q_{4} \rightarrow 0$ of these two last relations can also be recovered from the expressions given in eq. (3.25) of ref. [32].

\section{B The low-energy expansion of the three-point function $\mathcal{W}_{\mu \nu \rho}$}

In this appendix I reproduce the expressions of the invariant functions $w_{i}, i=0,1,2,3$, which provide a decomposition of the three-point function $\mathcal{W}^{\mu \nu \rho}$, obtained from a one-loop calculation in the low-energy expansion [57-59] with three light flavours [60]. For this, I also need the effective Lagrangian at order $\mathcal{O}\left(p^{6}\right)$ in the sector of odd intrinsic parity, whose general structure has been worked out in refs. [61,62]. For definiteness, I will adopt the basis of counter-terms given in the second of these two references. These expressions then allow to discuss, within this framework, a certain number of properties mentioned at various places in the main text. For reasons of simplicity, I only give the expressions corresponding to the iso-triplet channel. Similar expressions can be worked out in the octet channel. A discussion of the singlet channel would require to work within the framework 
of a combined chiral and $1 / N_{c}$ expansion, which is in principle also possible, since the necessary tools are available $[60,63,64]$.

At one loop in chiral perturbation theory, one obtains the following results

$$
\begin{aligned}
w_{1}^{3}\left(q_{1}^{2}, q_{2}^{2},\left(q_{1}+q_{2}\right)^{2}\right)= & \frac{N_{c}}{144 \pi^{2} F_{\pi}^{2}}\left[\left(1-\frac{4 M_{\pi}^{2}}{q_{1}^{2}}\right) \bar{J}_{\pi \pi}\left(q_{1}^{2}\right)-\left(1-\frac{4 M_{\pi}^{2}}{q_{2}^{2}}\right) \bar{J}_{\pi \pi}\left(q_{2}^{2}\right)\right] \\
& +\frac{N_{c}}{144 \pi^{2} F_{\pi}^{2}}\left[\left(1-\frac{4 M_{K}^{2}}{q_{1}^{2}}\right) \bar{J}_{K K}\left(q_{1}^{2}\right)-\left(1-\frac{4 M_{K}^{2}}{q_{2}^{2}}\right) \bar{J}_{K K}\left(q_{2}^{2}\right)\right] \\
& +\mathcal{O}\left(p^{8}\right), \\
w_{2}^{3}\left(q_{1}^{2}, q_{2}^{2},\left(q_{1}+q_{2}\right)^{2}\right)= & -\frac{16}{3} C_{22}^{W}(\mu)+\frac{N_{c}}{72 \pi^{2} F_{\pi}^{2}} \frac{1}{16 \pi^{2}}\left(\ln \frac{M_{\pi}^{2}}{\mu^{2}}+\ln \frac{M_{K}^{2}}{\mu^{2}}+\frac{2}{3}\right) \\
& -\frac{N_{c}}{144 \pi^{2} F_{\pi}^{2}}\left[\left(1-\frac{4 M_{\pi}^{2}}{q_{1}^{2}}\right) \bar{J}_{\pi \pi}\left(q_{1}^{2}\right)+\left(1-\frac{4 M_{\pi}^{2}}{q_{2}^{2}}\right) \bar{J}_{\pi \pi}\left(q_{2}^{2}\right)\right] \\
& -\frac{N_{c}}{144 \pi^{2} F_{\pi}^{2}}\left[\left(1-\frac{4 M_{K}^{2}}{q_{1}^{2}}\right) \bar{J}_{K K}\left(q_{1}^{2}\right)+\left(1-\frac{4 M_{K}^{2}}{q_{2}^{2}}\right) \bar{J}_{K K}\left(q_{2}^{2}\right)\right] \\
& +\mathcal{O}\left(p^{8}\right), \\
w_{3}^{3}\left(q_{1}^{2}, q_{2}^{2},\left(q_{1}+q_{2}\right)^{2}\right)= & -w_{1}^{3}\left(q_{1}^{2}, q_{2}^{2},\left(q_{1}+q_{2}\right)^{2}\right)+\mathcal{O}\left(p^{8}\right),
\end{aligned}
$$

and

$$
\begin{aligned}
\mathcal{H}^{3}\left(q_{1}^{2}, q_{2}^{2},\left(q_{1}+q_{2}\right)^{2}\right)= & \frac{64}{3} C_{7}^{W} M_{\pi}^{2}+\frac{M_{\pi}^{2} F_{\pi} \widehat{\mathcal{F}}_{\pi \gamma^{*} \gamma^{*}}\left(q_{1}^{2}, q_{2}^{2}\right)}{\left(q_{1}+q_{2}\right)^{2}-M_{\pi}^{2}}+\mathcal{O}\left(p^{8}\right), \\
F_{\pi} \widehat{\mathcal{F}}_{\pi \gamma^{*} \gamma^{*}}\left(q_{1}^{2}, q_{2}^{2}\right)= & \mathcal{A}^{3}+\frac{64}{3} C_{7}^{W} M_{\pi}^{2} \\
& +\left[-\frac{16}{3} C_{22}^{W}(\mu)+\frac{N_{c}}{72 \pi^{2} F_{\pi}^{2}} \frac{1}{16 \pi^{2}}\left(\ln \frac{M_{\pi}^{2}}{\mu^{2}}+\ln \frac{M_{K}^{2}}{\mu^{2}}+\frac{2}{3}\right)\right]\left(q_{1}^{2}+q_{2}^{2}\right) \\
& -\frac{N_{c}}{72 \pi^{2} F_{\pi}^{2}}\left[\left(q_{1}^{2}-4 M_{\pi}^{2}\right) \bar{J}_{\pi \pi}\left(q_{1}^{2}\right)+\left(q_{2}^{2}-4 M_{\pi}^{2}\right) \bar{J}_{\pi \pi}\left(q_{2}^{2}\right)\right] \\
& -\frac{N_{c}}{72 \pi^{2} F_{\pi}^{2}}\left[\left(q_{1}^{2}-4 M_{\pi}^{2}\right) \bar{J}_{K K}\left(q_{1}^{2}\right)+\left(q_{2}^{2}-4 M_{\pi}^{2}\right) \bar{J}_{K K}\left(q_{2}^{2}\right)\right]+\mathcal{O}\left(p^{8}\right),
\end{aligned}
$$

where $\mathcal{A}^{3}=-N_{c} / 12 \pi^{2}=(3 / 4) \mathcal{A}$ and the loop function $\bar{J}_{P P}, P=\pi, K$, is defined in ref. [60] and can be conveniently expressed as the integral

$$
\bar{J}_{P P}(s)=-\frac{1}{16 \pi^{2}} \int_{0}^{1} d u \ln \left[1-\frac{s}{M_{P}^{2}} u(1-u)\right] .
$$

Furthermore, $\mu$ denotes the chiral renormalization scale. The low-energy constant $C_{7}^{W}$ is $\mu$ independent, while the $\mu$-dependence of the renormalized constant $C_{22}^{W}(\mu)$ is compensated by the $\log \mu^{2}$ terms, see ref. [62]. Notice that despite the suggestive notation, and as the - symbol is meant to remind of, $\widehat{\mathcal{F}}_{\pi \gamma^{*} \gamma^{*}}\left(q_{1}^{2}, q_{2}^{2}\right)$ is not yet the pion transition form factor $\mathcal{F}_{\pi \gamma^{*} \gamma^{*}}\left(q_{1}^{2}, q_{2}^{2}\right)$. The relation between the two is given by

$$
\mathcal{F}_{\pi \gamma^{*} \gamma^{*}}\left((p / 2+q)^{2},(p / 2-q)^{2}\right)=\lim _{p^{2} \rightarrow M_{\pi}^{2}} \widehat{\mathcal{F}}_{\pi \gamma^{*} \gamma^{*}}\left((p / 2+q)^{2},(p / 2-q)^{2}\right) .
$$


In the semi-off-shell case the expression of $\mathcal{F}_{\pi \gamma^{*} \gamma^{*}}\left((p / 2 \pm q)^{2}, 0\right)$ one obtains this way reproduces the one that is given in ref. [65]. From these formulas, one deduces, through the relations given in eq. (2.34), the one-loop expression of the remaining functions

$$
w_{0}^{3}\left(q_{1}^{2}, q_{2}^{2},\left(q_{1}+q_{2}\right)^{2}\right)=-w_{2}^{3}\left(q_{1}^{2}, q_{2}^{2},\left(q_{1}+q_{2}\right)^{2}\right)+\frac{F_{\pi} \widehat{\mathcal{F}}_{\pi \gamma^{*} \gamma^{*}}\left(q_{1}^{2}, q_{2}^{2}\right)}{\left(q_{1}+q_{2}\right)^{2}-M_{\pi}^{2}}+\mathcal{O}\left(p^{8}\right),
$$

and

$$
\begin{aligned}
\frac{1}{8 \pi^{2}} w_{L}^{3}\left(q_{1}^{2}, q_{2}^{2},\left(q_{1}+q_{2}\right)^{2}\right)= & \frac{1}{\left(q_{1}+q_{2}\right)^{2}}\left[\mathcal{A}^{3}+\frac{64}{3} C_{7}^{W} M_{\pi}^{2}+\frac{M_{\pi}^{2} F_{\pi} \widehat{\mathcal{F}}_{\pi \gamma^{*} \gamma^{*}}\left(q_{1}^{2}, q_{2}^{2}\right)}{\left(q_{1}+q_{2}\right)^{2}-M_{\pi}^{2}}\right]+\mathcal{O}\left(p^{8}\right) \\
= & \frac{1}{\left(q_{1}+q_{2}\right)^{2}}\left[F_{\pi} \widehat{\mathcal{F}}_{\pi \gamma^{*} \gamma^{*}}(0,0)+\frac{M_{\pi}^{2} F_{\pi} \widehat{\mathcal{F}}_{\pi \gamma^{*} \gamma^{*}}\left(q_{1}^{2}, q_{2}^{2}\right)}{\left(q_{1}+q_{2}\right)^{2}-M_{\pi}^{2}}\right]+\mathcal{O}\left(p^{8}\right) \\
= & \frac{F_{\pi} \widehat{\mathcal{F}}_{\pi \gamma^{*} \gamma^{*}}(0,0)}{\left(q_{1}+q_{2}\right)^{2}-M_{\pi}^{2}}+\frac{M_{\pi}^{2}}{\left(q_{1}+q_{2}\right)^{2}} \frac{F_{\pi}\left[\widehat{\mathcal{F}}_{\pi \gamma^{*} \gamma^{*}}\left(q_{1}^{2}, q_{2}^{2}\right)-\widehat{\mathcal{F}}_{\pi \gamma^{*} \gamma^{*}}(0,0)\right]}{\left(q_{1}+q_{2}\right)^{2}-M_{\pi}^{2}} \\
& +\mathcal{O}\left(p^{8}\right) .
\end{aligned}
$$

The kinematic singularity, at $\left(q_{1}+q_{2}\right)^{2}=0$, of $w_{L}^{3}$ is immediately visible in this expression. It also shows how, in the chiral limit, this kinematic singularity transforms into a dynamical singularity due to the massless pion pole, but with a constant residue, fixed by the anomaly,

$$
\lim _{m_{q} \rightarrow 0} F_{\pi} \widehat{\mathcal{F}}_{\pi \gamma^{*} \gamma^{*}}(0,0)=\mathcal{A}^{3}
$$

The combination that appears in the short-distance condition (3.4) for $\hat{\mathcal{W}}_{1}$ is completely different already at one loop, since

$$
\begin{aligned}
w_{0}^{3}\left(q_{1}^{2}, q_{2}^{2},\left(q_{1}+q_{2}\right)^{2}\right)+w_{1}^{3}\left(q_{1}^{2}, q_{2}^{2},\left(q_{1}+q_{2}\right)^{2}\right)= & \frac{F_{\pi} \widehat{\mathcal{F}}_{\pi \gamma^{*} \gamma^{*}}\left(q_{1}^{2}, q_{2}^{2}\right)}{\left(q_{1}+q_{2}\right)^{2}-M_{\pi}^{2}} \\
& +\frac{16}{3} C_{22}^{W}(\mu) \\
& -\frac{N_{c}}{72 \pi^{2} F_{\pi}^{2}} \frac{1}{16 \pi^{2}}\left(\ln \frac{M_{\pi}^{2}}{\mu^{2}}+\ln \frac{M_{K}^{2}}{\mu^{2}}+\frac{2}{3}\right) \\
& +\frac{N_{c}}{72 \pi^{2} F_{\pi}^{2}}\left(1-\frac{4 M_{\pi}^{2}}{q_{1}^{2}}\right) \bar{J}_{\pi \pi}\left(q_{1}^{2}\right) \\
& +\frac{N_{c}}{72 \pi^{2} F_{\pi}^{2}}\left(1-\frac{4 M_{K}^{2}}{q_{1}^{2}}\right) \bar{J}_{K K}\left(q_{1}^{2}\right)+\mathcal{O}\left(p^{8}\right) .
\end{aligned}
$$

As stated in the text, it exhibits a pion pole, with residue given by $F_{\pi} \mathcal{F}_{\pi \gamma^{*} \gamma^{*}}\left(q_{1}^{2}, q_{2}^{2}\right)$ that retains a non-trivial momentum dependence even in the chiral limit. The difference between 
the two expressions can be given a suggestive form,

$$
\begin{gathered}
w_{0}^{3}\left(q_{1}^{2}, q_{2}^{2},\left(q_{1}+q_{2}\right)^{2}\right)+w_{1}^{3}\left(q_{1}^{2}, q_{2}^{2},\left(q_{1}+q_{2}\right)^{2}\right)-\frac{1}{8 \pi^{2}} w_{L}^{3}\left(q_{1}^{2}, q_{2}^{2},\left(q_{1}+q_{2}\right)^{2}\right) \\
=\left[1-\frac{q_{1}^{2}}{\left(q_{1}+q_{2}\right)^{2}}\right]\left\{\frac{16}{3} C_{22}^{W}(\mu)-\frac{N_{c}}{72 \pi^{2} F_{\pi}^{2}} \frac{1}{16 \pi^{2}}\left(\ln \frac{M_{\pi}^{2}}{\mu^{2}}+\ln \frac{M_{K}^{2}}{\mu^{2}}+\frac{2}{3}\right)\right. \\
\left.\quad+\frac{N_{c}}{72 \pi^{2} F_{\pi}^{2}}\left(1-\frac{4 M_{\pi}^{2}}{q_{1}^{2}}\right) \bar{J}_{\pi \pi}\left(q_{1}^{2}\right)+\frac{N_{c}}{72 \pi^{2} F_{\pi}^{2}}\left(1-\frac{4 M_{K}^{2}}{q_{1}^{2}}\right) \bar{J}_{K K}\left(q_{1}^{2}\right)\right\} \\
-\frac{q_{2}^{2}}{\left(q_{1}+q_{2}\right)^{2}}\left\{\frac{16}{3} C_{22}^{W}(\mu)-\frac{N_{c}}{72 \pi^{2} F_{\pi}^{2}} \frac{1}{16 \pi^{2}}\left(\ln \frac{M_{\pi}^{2}}{\mu^{2}}+\ln \frac{M_{K}^{2}}{\mu^{2}}+\frac{2}{3}\right)\right. \\
\left.+\frac{N_{c}}{72 \pi^{2} F_{\pi}^{2}}\left(1-\frac{4 M_{\pi}^{2}}{q_{2}^{2}}\right) \bar{J}_{\pi \pi}\left(q_{2}^{2}\right)+\frac{N_{c}}{72 \pi^{2} F_{\pi}^{2}}\left(1-\frac{4 M_{K}^{2}}{q_{2}^{2}}\right) \bar{J}_{K K}\left(q_{2}^{2}\right)\right\}+\mathcal{O}\left(p^{8}\right) .
\end{gathered}
$$

It clearly exhibits the cancellation that takes place in the limit $q_{2} \rightarrow 0$. The corresponding expressions in the chiral limit $m_{q} \rightarrow 0$ can be easily worked out from the formulas given above, using

$$
\lim _{M_{P} \rightarrow 0}\left[\bar{J}_{P P}(s)-\frac{1}{16 \pi^{2}} \ln \frac{M_{P}^{2}}{\mu^{2}}\right]=-\frac{1}{16 \pi^{2}} \ln \frac{-s}{\mu^{2}}+\frac{1}{8 \pi^{2}} .
$$

Whether one then takes the limit where the four-vector $q_{2}$ vanishes, or the combined, "dispersive-friendly", limit $q_{2}^{2} \rightarrow 0,\left(q_{1}+q_{2}\right)^{2} \rightarrow q_{1}^{2}$, one obtains the same result,

$$
\begin{aligned}
\lim _{q_{2} \rightarrow 0} \lim _{m_{q} \rightarrow 0}\left[w_{0}^{3}\left(q_{1}^{2}, q_{2}^{2},\left(q_{1}+q_{2}\right)^{2}\right)+w_{1}^{3}\left(q_{1}^{2}, q_{2}^{2},\left(q_{1}+q_{2}\right)^{2}\right)\right] & =\lim _{q_{2} \rightarrow 0 m_{q} \rightarrow 0} \lim _{8 \pi^{2}} \frac{1}{8} w_{L}^{3}\left(q_{1}^{2}, q_{2}^{2},\left(q_{1}+q_{2}\right)^{2}\right) \\
& =\frac{\mathcal{A}^{3}}{q_{1}^{2}} .
\end{aligned}
$$

But the manner how this result comes about is totally different in the two cases. To see this in an easy manner, let me consider the combined chiral and large- $N_{c}$ limit, where one finds the simple expressions [in the large- $N_{c}$ limit, $L_{22}^{W}$ scales as $\mathcal{O}\left(N_{c}\right)$ and becomes independent of the renormalization scale $\mu$, and recall that $\mathcal{A}^{3}$ is also proportional to $N_{c}$, whereas $F_{\pi}$ scales as $\left.\mathcal{O}\left(\sqrt{N_{c}}\right)\right]$

$$
\begin{aligned}
\lim _{\substack{m_{q} \rightarrow 0 \\
N_{c} \rightarrow \infty}} F_{\pi} \widehat{\mathcal{F}}_{\pi \gamma^{*} \gamma^{*}}\left(q_{1}^{2}, q_{2}^{2}\right) & =\mathcal{A}^{3}-\frac{16}{3} C_{22}^{W}\left(q_{1}^{2}+q_{2}^{2}\right)+\mathcal{O}\left(p^{6} N_{c}^{0}, p^{8} N_{c}\right) \\
\lim _{\substack{m_{q} \rightarrow 0 \\
N_{c} \rightarrow \infty}}\left[w_{0}^{3}\left(q_{1}^{2}, q_{2}^{2},\left(q_{1}+q_{2}\right)^{2}\right)+w_{1}^{3}\left(q_{1}^{2}, q_{2}^{2},\left(q_{1}+q_{2}\right)^{2}\right)\right]= & \frac{\mathcal{A}^{3}-(16 / 3) C_{22}^{W}\left(q_{1}^{2}+q_{2}^{2}\right)}{\left(q_{1}+q_{2}\right)^{2}}+\frac{16}{3} C_{22}^{W} \\
& +\mathcal{O}\left(p^{6} N_{c}^{0}, p^{8} N_{c}\right), \\
\lim _{\substack{m_{q} \rightarrow 0 \\
N_{c} \rightarrow \infty}} w_{L}^{3}\left(q_{1}^{2}, q_{2}^{2},\left(q_{1}+q_{2}\right)^{2}\right) & =\frac{\mathcal{A}^{3}}{\left(q_{1}+q_{2}\right)^{2}} .
\end{aligned}
$$

As is well known, there are no corrections to the above expression of $w_{L}^{3}\left(q_{1}^{2}, q_{2}^{2},\left(q_{1}+q_{2}\right)^{2}\right)$ in the chiral limit $[66,67]$. In the case of $w_{L}^{3}$, it is straightforward to understand how the limit in eq. (B.11) arises. It simply reflects the fact that in the limit under consideration all that survives is the kinematic pole that has actually become a dynamical pion pole, with constant residue fixed by the anomaly, and there is nothing else, even before the limit $q_{2} \rightarrow 0$ 
is taken, as shown in the last expression in eq. (B.12). In the case of the sum $w_{0}^{3}+w_{1}^{3}$, the situation is somewhat more subtle. There are other contributions besides a pion pole with constant residue in eq. (B.12) before the limit $q_{2} \rightarrow 0$ is taken: the momentum-dependent residue of the pole is given by whatever is left over from $F_{\pi} \mathcal{F}_{\pi \gamma^{*} \gamma^{*}}\left(q_{1}^{2}, q_{2}^{2}\right)$ in the combined chiral and large- $N_{c}$ limit, i.e. here a contribution proportional to $C_{22}^{W}$, and there are other, non-pole, contributions, also proportional to $C_{22}^{W}$. When the limit $q_{2} \rightarrow 0$ is taken, these two different contributions combine such as to leave only a part of the full pion pole, the one with a constant residue $F_{0} \stackrel{\mathrm{o}}{\mathcal{F}}_{\pi \gamma^{*} \gamma^{*}}(0,0)=\mathcal{A}^{3}$, behind. That this will happen that way to higher, and in fact, to all orders in the low-energy expansion, is guaranteed by eq. (2.34), so that eq. (B.11) actually constitutes an exact result of QCD. But as far as $w_{0}^{3}+w_{1}^{3}$ is concerned, it only reproduces a truncated part of the full pion pole that was present to start with. In a nutshell, sometimes the two operations of taking the limit $q_{2} \rightarrow 0$ and of extracting the pion pole do not commute.

Open Access. This article is distributed under the terms of the Creative Commons Attribution License (CC-BY 4.0), which permits any use, distribution and reproduction in any medium, provided the original author(s) and source are credited.

\section{References}

[1] Muon G-2 collaboration, Final report of the muon E821 anomalous magnetic moment measurement at BNL, Phys. Rev. D 73 (2006) 072003 [hep-ex/0602035] [INSPIRE].

[2] F. Jegerlehner, Muon g-2 theory: the hadronic part, EPJ Web Conf. 166 (2018) 00022 [arXiv: 1705.00263] [INSPIRE].

[3] M. Davier, A. Hoecker, B. Malaescu and Z. Zhang, A new evaluation of the hadronic vacuum polarisation contributions to the muon anomalous magnetic moment and to $\alpha\left(m_{Z}^{2}\right)$, Eur. Phys. J. C 80 (2020) 241 [Erratum ibid. 80 (2020) 410] [arXiv: 1908. 00921] [INSPIRE].

[4] A. Keshavarzi, D. Nomura and T. Teubner, $g-2$ of charged leptons, $\alpha\left(M_{Z}^{2}\right)$, and the hyperfine splitting of muonium, Phys. Rev. D 101 (2020) 014029 [arXiv:1911.00367] [INSPIRE].

[5] M. Della Morte et al., The hadronic vacuum polarization contribution to the muon $g-2$ from lattice QCD, JHEP 10 (2017) 020 [arXiv:1705.01775] [INSPIRE].

[6] RBC and UKQCD collaborations, Calculation of the hadronic vacuum polarization contribution to the muon anomalous magnetic moment, Phys. Rev. Lett. 121 (2018) 022003 [arXiv: 1801.07224] [inSPIRE].

[7] D. Giusti, V. Lubicz, G. Martinelli, F. Sanfilippo and S. Simula, Electromagnetic and strong isospin-breaking corrections to the muon $g-2$ from lattice $Q C D+Q E D$, Phys. Rev. D 99 (2019) 114502 [arXiv: 1901.10462] [INSPIRE].

[8] Fermilab Lattice, LATTICE-HPQCD and MiLC collaborations, Hadronic-vacuum-polarization contribution to the muon's anomalous magnetic moment from four-flavor lattice QCD, Phys. Rev. D 101 (2020) 034512 [arXiv:1902.04223] [INSPIRE].

[9] A. Gérardin et al., The leading hadronic contribution to $(g-2)_{\mu}$ from lattice $Q C D$ with $N_{\mathrm{f}}=2+1$ flavours of $O(a)$ improved Wilson quarks, Phys. Rev. D 100 (2019) 014510 [arXiv: 1904.03120] [INSPIRE]. 
[10] S. Borsányi et al., Leading-order hadronic vacuum polarization contribution to the muon magnetic momentfrom lattice QCD, arXiv:2002.12347 [INSPIRE].

[11] G. Abbiendi, Letter of intent: the MUonE project, Tech. Rep. CERN-SPSC-2019-026, CERN, Geneva, Switzerland, 5 June 2019 [SPSC-I-252].

[12] C.M. Carloni Calame, M. Passera, L. Trentadue and G. Venanzoni, A new approach to evaluate the leading hadronic corrections to the muon g-2, Phys. Lett. B 746 (2015) 325 [arXiv: 1504.02228] [INSPIRE].

[13] G. Abbiendi et al., Measuring the leading hadronic contribution to the muon $g-2$ via $\mu$ e scattering, Eur. Phys. J. C 77 (2017) 139 [arXiv:1609.08987] [INSPIRE].

[14] P. Banerjee et al., Theory for muon-electron scattering @10 ppm: a report of the MUonE theory initiative, Eur. Phys. J. C 80 (2020) 591 [arXiv:2004.13663] [INSPIRE].

[15] T. Aoyama et al., The anomalous magnetic moment of the muon in the Standard Model, arXiv: 2006.04822 [INSPIRE].

[16] G. 't Hooft, A planar diagram theory for strong interactions, Nucl. Phys. B 72 (1974) 461 [INSPIRE].

[17] E. Witten, Baryons in the 1/n expansion, Nucl. Phys. B 160 (1979) 57 [INSPIRE].

[18] E. de Rafael, Hadronic contributions to the muon $g-2$ and low-energy QCD, Phys. Lett. B 322 (1994) 239 [hep-ph/9311316] [INSPIRE].

[19] M. Knecht, A. Nyffeler, M. Perrottet and E. de Rafael, Hadronic light by light scattering contribution to the muon $g-2$ : an effective field theory approach, Phys. Rev. Lett. 88 (2002) 071802 [hep-ph/0111059] [INSPIRE].

[20] M. Hayakawa, T. Kinoshita and A.I. Sanda, Hadronic light by light scattering effect on muon g-2, Phys. Rev. Lett. 75 (1995) 790 [hep-ph/9503463] [INSPIRE].

[21] J. Bijnens, E. Pallante and J. Prades, Hadronic light by light contributions to the muon $g-2$ in the large $N_{c}$ limit, Phys. Rev. Lett. 75 (1995) 1447 [Erratum ibid. 75 (1995) 3781] [hep-ph/9505251] [INSPIRE].

[22] J. Bijnens, E. Pallante and J. Prades, Analysis of the hadronic light by light contributions to the muon g-2, Nucl. Phys. B 474 (1996) 379 [hep-ph/9511388] [INSPIRE].

[23] M. Hayakawa, T. Kinoshita and A.I. Sanda, Hadronic light by light scattering contribution to muon g-2, Phys. Rev. D 54 (1996) 3137 [hep-ph/9601310] [InSPIRE].

[24] M. Hayakawa and T. Kinoshita, Pseudoscalar pole terms in the hadronic light by light scattering contribution to muon g-2, Phys. Rev. D 57 (1998) 465 [Erratum ibid. 66 (2002) 019902] [hep-ph/9708227] [INSPIRE].

[25] M. Hayakawa and T. Kinoshita, Comment on the sign of the pseudoscalar pole contribution to the muon $g-2$, hep-ph/0112102 [INSPIRE].

[26] J. Bijnens, E. Pallante and J. Prades, Comment on the pion pole part of the light by light contribution to the muon $g-2$, Nucl. Phys. B 626 (2002) 410 [hep-ph/0112255] [INSPIRE].

[27] J. Prades, The Standard Model prediction for muon $g-2$, in KAON2001: international conference on CP-violation, (2001) [hep-ph/0108192] [INSPIRE].

[28] M. Knecht and A. Nyffeler, Hadronic light by light corrections to the muon $g-2$ : the pion pole contribution, Phys. Rev. D 65 (2002) 073034 [hep-ph/0111058] [INSPIRE]. 
[29] A. Nyffeler, Hadronic light-by-light scattering in the muon g-2: a new short-distance constraint on pion-exchange, Phys. Rev. D 79 (2009) 073012 [arXiv:0901.1172] [INSPIRE].

[30] F. Jegerlehner and A. Nyffeler, The muon g-2, Phys. Rept. 477 (2009) 1 [arXiv:0902.3360] [INSPIRE].

[31] G. Colangelo, F. Hagelstein, M. Hoferichter, L. Laub and P. Stoffer, Short-distance constraints on hadronic light-by-light scattering in the anomalous magnetic moment of the muon, Phys. Rev. D 101 (2020) 051501 [arXiv:1910.11881] [INSPIRE].

[32] G. Colangelo, F. Hagelstein, M. Hoferichter, L. Laub and P. Stoffer, Longitudinal short-distance constraints for the hadronic light-by-light contribution to $(g-2)_{\mu}$ with large- $N_{c}$ Regge models, JHEP 03 (2020) 101 [arXiv:1910.13432] [INSPIRE].

[33] K. Melnikov and A. Vainshtein, On dispersion relations and hadronic light-by-light scattering contribution to the muon anomalous magnetic moment, arXiv:1911.05874 [INSPIRE].

[34] K. Melnikov and A. Vainshtein, Hadronic light-by-light scattering contribution to the muon anomalous magnetic moment revisited, Phys. Rev. D 70 (2004) 113006 [hep-ph/0312226] [INSPIRE].

[35] J. Aldins, T. Kinoshita, S.J. Brodsky and A.J. Dufner, Photon-photon scattering contribution to the sixth order magnetic moments of the muon and electron, Phys. Rev. D 1 (1970) 2378 [INSPIRE].

[36] G. Colangelo, M. Hoferichter, M. Procura and P. Stoffer, Dispersion relation for hadronic light-by-light scattering: theoretical foundations, JHEP 09 (2015) 074 [arXiv:1506.01386] [INSPIRE].

[37] J.D. Bjorken, Applications of the chiral $\mathrm{U}(6) \times \mathrm{U}(6)$ algebra of current densities, Phys. Rev. 148 (1966) 1467 [INSPIRE].

[38] K. Johnson and F.E. Low, Current algebras in a simple model, Prog. Theor. Phys. Suppl. 37 (1966) 74 [INSPIRE].

[39] J. Bijnens, N. Hermansson-Truedsson and A. Rodríguez-Sánchez, Short-distance constraints for the HLbL contribution to the muon anomalous magnetic moment, Phys. Lett. B 798 (2019) 134994 [arXiv: 1908.03331] [INSPIRE].

[40] S.L. Adler, Axial vector vertex in spinor electrodynamics, Phys. Rev. 177 (1969) 2426 [INSPIRE].

[41] J.S. Bell and R. Jackiw, A PCAC puzzle: $\pi^{0} \rightarrow \gamma \gamma$ in the $\sigma$ model, Nuovo Cim. A 60 (1969) 47 [INSPIRE].

[42] L. Rosenberg, Electromagnetic interactions of neutrinos, Phys. Rev. 129 (1963) 2786 [INSPIRE].

[43] R. Jackiw, Field theoretic investigations in current algebra, in Current algebra and anomalies, S.B. Treiman, E. Witten, R. Jackiw and B. Zumino, World Scientific, Singapore (1985), pg. 81.

[44] M. Knecht, S. Peris, M. Perrottet and E. de Rafael, New nonrenormalization theorems for anomalous three point functions, JHEP 03 (2004) 035 [hep-ph/0311100] [INSPIRE].

[45] D.G. Sutherland, Current algebra and some nonstrong mesonic decays, Nucl. Phys. B 2 (1967) 433 [INSPIRE]. 
[46] M.J.G. Veltman, I. Theoretical aspects of high energy neutrino interactions, Proc. Roy. Soc. London A 301 (1967) 107.

[47] G.M. Shore and G. Veneziano, The polarized photon structure function $g_{1}^{\gamma}$ as a probe of chiral symmetry realizations, Mod. Phys. Lett. A 8 (1993) 373 [INSPIRE].

[48] P. Roig and P. Sanchez-Puertas, Axial-vector exchange contribution to the hadronic light-by-light piece of the muon anomalous magnetic moment, Phys. Rev. D 101 (2020) 074019 [arXiv: 1910.02881] [INSPIRE].

[49] G. Colangelo, M. Hoferichter, M. Procura and P. Stoffer, Dispersion relation for hadronic light-by-light scattering: two-pion contributions, JHEP 04 (2017) 161 [arXiv:1702.07347] [INSPIRE].

[50] S. Weinberg, High-energy behavior in quantum field theory, Phys. Rev. 118 (1960) 838 [INSPIRE].

[51] V.A. Novikov, M.A. Shifman, A.I. Vainshtein, M.B. Voloshin and V.I. Zakharov, Use and misuse of QCD sum rules, factorization and related topics, Nucl. Phys. B 237 (1984) 525 [INSPIRE].

[52] V.A. Nesterenko and A.V. Radyushkin, Comparison of the QCD sum rule approach and perturbative QCD analysis for $\gamma^{*} \gamma^{*} \rightarrow \pi^{0}$ process, Sov. J. Nucl. Phys. 38 (1983) 284 [Yad. Fiz. 38 (1983) 476] [INSPIRE].

[53] G. Lepage and S.J. Brodsky, Exclusive processes in quantum chromodynamics: evolution equations for hadronic wave functions and the form-factors of mesons, Phys. Lett. B 87 (1979) 359 [INSPIRE].

[54] G. Lepage and S.J. Brodsky, Exclusive processes in perturbative quantum chromodynamics, Phys. Rev. D 22 (1980) 2157 [INSPIRE].

[55] K. Kampf and J. Novotny, Resonance saturation in the odd-intrinsic parity sector of low-energy QCD, Phys. Rev. D 84 (2011) 014036 [arXiv:1104.3137] [InSPIRE].

[56] P. Masjuan, P. Roig and P. Sanchez-Puertas, A different viewpoint on the hadronic light-by-light tensor short-distance constraints, arXiv:2005.11761 [INSPIRE].

[57] S. Weinberg, Phenomenological Lagrangians, Physica A 96 (1979) 327 [InSPIRE].

[58] J. Gasser and H. Leutwyler, On the low-energy structure of QCD, Phys. Lett. B 125 (1983) 321 [INSPIRE].

[59] J. Gasser and H. Leutwyler, Chiral perturbation theory to one loop, Annals Phys. 158 (1984) 142 [INSPIRE].

[60] J. Gasser and H. Leutwyler, Chiral perturbation theory: expansions in the mass of the strange quark, Nucl. Phys. B 250 (1985) 465 [INSPIRE].

[61] T. Ebertshauser, H.W. Fearing and S. Scherer, The anomalous chiral perturbation theory meson Lagrangian to order $p^{6}$ revisited, Phys. Rev. D 65 (2002) 054033 [hep-ph/0110261] [INSPIRE].

[62] J. Bijnens, L. Girlanda and P. Talavera, The anomalous chiral Lagrangian of order $p^{6}$, Eur. Phys. J. C 23 (2002) 539 [hep-ph/0110400] [INSPIRE].

[63] P. Herrera-Siklody, J.I. Latorre, P. Pascual and J. Taron, Chiral effective Lagrangian in the large $N_{c}$ limit: the nonet case, Nucl. Phys. B 497 (1997) 345 [hep-ph/9610549] [InSPIRE]. 
[64] R. Kaiser and H. Leutwyler, Large $N_{c}$ in chiral perturbation theory, Eur. Phys. J. C 17 (2000) 623 [hep-ph/0007101] [INSPIRE].

[65] J. Bijnens, A. Bramon and F. Cornet, Pseudoscalar decays into photon-photon in chiral perturbation theory, Phys. Rev. Lett. 61 (1988) 1453 [INSPIRE].

[66] S.L. Adler and W.A. Bardeen, Absence of higher order corrections in the anomalous axial vector divergence equation, Phys. Rev. 182 (1969) 1517 [INSPIRE].

[67] E. Witten, Global aspects of current algebra, Nucl. Phys. B 223 (1983) 422 [InSPIRE]. 